\title{
Mutant Cullin 3 causes familial hyperkalemic hypertension via dominant effects
}

\author{
Mohammed Z. Ferdaus, ${ }^{1}$ Lauren N. Miller, ${ }^{1}$ Larry N. Agbor, ${ }^{2}$ Turgay Saritas, ${ }^{1}$ Jeffrey D. Singer, ${ }^{3}$ \\ Curt D. Sigmund, ${ }^{2,4}$ and James A. McCormick ${ }^{1}$ \\ 'Division of Nephrology \& Hypertension, Department of Medicine, Oregon Health \& Science University, Portland, Oregon, \\ USA. 'Department of Pharmacology, Roy J. and Lucille A. Carver College of Medicine, University of lowa, lowa City, lowa, \\ USA. ${ }^{3}$ Department of Biology, Portland State University, Portland, Oregon, USA. ${ }^{4}$ UIHC Center for Hypertension Research, \\ Roy J. and Lucille A. Carver College of Medicine, University of lowa, lowa City, lowa, USA.
}

Mutations in the ubiquitin ligase scaffold protein Cullin 3 (CUL3) cause the disease familial hyperkalemic hypertension (FHHt). In the kidney, mutant CUL3 (CUL3- $\Delta 9$ ) increases abundance of With-No-Lysine [K] Kinase 4 (WNK4), with excessive activation of the downstream Sterile 20 (STE20)/SPS-1-related proline/alanine-rich kinase (SPAK) increasing phosphorylation of the $\mathrm{Na}^{+}-$ $\mathrm{Cl}^{-}$cotransporter (NCC). CUL3- $\Delta 9$ promotes its own degradation via autoubiquitination, leading to the hypothesis that Cul3 haploinsufficiency causes FHHt. To directly test this, we generated Cul3 heterozygous mice (CUL3-Het), and Cul3 heterozygotes also expressing CUL3- $\Delta \mathbf{9}$ (CUL3-Het/ $\Delta 9)$, using an inducible renal epithelial-specific system. Endogenous CUL3 was reduced to $50 \%$ in both models, and consistent with autoubiquitination, CUL3- $\Delta 9$ protein was undetectable in CUL3-Het/ $\Delta 9$ kidneys unless primary renal epithelia cells were cultured. Abundances of WNK4 and phosphorylated NCC did not differ between control and CUL3-Het mice, but they were elevated in CUL3-Het/ $\Delta 9$ mice, which also displayed higher plasma $\left[\mathrm{K}^{+}\right]$and blood pressure. Abundance of phosphorylated $\mathrm{Na}^{+}-\mathrm{K}^{+}-2 \mathrm{Cl}^{-}$cotransporter (NKCC2) was also increased, which may contribute to the severity of CUL3- $\Delta$ 9-mediated FHHt. WNK4 and SPAK localized to puncta in NCC-positive segments but not in NKCC2-positive segments, suggesting differential effects of CUL3- $\Delta$ 9. These results indicate that $\mathrm{Cul} 3$ haploinsufficiency does not cause $\mathrm{FHHt}$, but dominant effects of CUL3- $\Delta 9$ are required.

Conflict of interest: The authors have declared that no conflict of interest exists.

Submitted: August 4, 2017 Accepted: November 15, 2017 Published: December 21, 2017

\section{Reference information:} JCI Insight. 2017;2(24):e96700 https://doi.org/10.1172/jici. insight.96700.

\section{Introduction}

Identifying the genes responsible for rare inherited diseases has led to profound insights into both normal and abnormal physiological processes. In the kidney, the disease familial hyperkalemic hypertension (FHHt) exemplifies how studying such genes can lead to the elucidation of novel regulatory pathways. The discovery that mutations in the With-no-lysine [K] kinases (WNKs) Wnk1 and Wnk4 cause FHHt (1, 2) and, more recently, that mutations in Cullin 3 (Cul3) (OMIM 614496, PHA2E) (3-5) and Kelch-like $3(\operatorname{Klhl3})(4,5)$ - components of an E3 ubiquitin ligase complex - also cause the disease has revealed a canonical pathway for the regulation of the $\mathrm{Na}^{+} \mathrm{Cl}^{-}$cotransporter (NCC). Along the distal convoluted tubule (DCT), NCC is activated by phosphorylation at several amino-terminal residues $(6,7)$. Rather than being directly phosphorylated by WNKs, phosphorylation is performed primarily by Sterile 20 (STE20)/SPS-1-related proline/alanine-rich kinase (SPAK) $(8,9)$, with Oxidative stress-response kinase-1 (OSR1) also playing a minor role (6). These kinases are activated by WNK-mediated phosphorylation. FHHt-causing mutations in $W n k 1$ or $W n k 4$ increase abundances of WNK1 and WNK4 protein, increasing phosphorylation and activation of SPAK/OSR1 $(1,2,10)$ and, hence, NCC phosphorylation. Increased NCC activity causes excessive $\mathrm{Na}^{+}$reabsorption that raises extracellular fluid volume and, thus, blood pressure. It has also been proposed to cause hyperkalemia by reducing $\mathrm{Na}^{+}$delivery to the epithelial sodium channel $(\mathrm{ENaC})$ along the collecting duct $(\mathrm{CD})$, which decreases the electrogenic drive for $\mathrm{K}^{+}$secretion through the renal outer medullary $\mathrm{K}^{+}$channel (ROMK) (11-15). However, it should be noted that direct effects of $W n k$ mutations on $\mathrm{Na}^{+}-\mathrm{K}^{+}-2 \mathrm{Cl}^{-}$cotransporter $(\mathrm{NKCC})(16), \mathrm{ENaC}(16,17)$, 
and ROMK $(16,18,19)$ may also play important roles in the etiology of FHHt. Wnk1 mutations are intronic and result in aberrant expression of WNK1 along the distal nephron (20), whereas Wnk4 mutations affect interactions between WNK4 and CUL3 and KLHL3.

CUL3 and KLHL3 are components of a complex (Cullin RING ligase, CRL) that mediates ubiquitination and proteasomal degradation of multiple targets. In the CRL, CUL3 acts as a scaffolding-protein, while KLHL3 serves as a substrate-binding adaptor $(21,22)$. Since the discovery that mutations in Cul3 and Klhl3 cause FHHt, it has been determined that both WNK1 and WNK4 are substrates for CUL3/ KLHL3-mediated proteasomal degradation (23-25). Ultimately, the CRL modulates NCC phosphorylation by regulating abundance of $\operatorname{WNK} 1$ and $\operatorname{WNK} 4(25,26)$; in FHHt, this is disrupted, leading to increased NCC activity. Mutations in Klhl3 prevent degradation of WNK4 by disrupting interactions between KLHL3 and its scaffold CUL3, or with WNK4 (26). Wnk4 mutations prevent WNK4 degradation, since they occur in a motif required for interaction with $\operatorname{KLHL3}(23,26)$. Mutations in $W n k 1$ that affect WNK1 degradation by the CRL have not been reported, but KLHL3-KO mice display increased WNK1 abundance (27). Mutations in Cul3 are autosomal dominant and cause the most severe FHHt phenotype (4). These mutations occur at splice sites leading to the generation of an in-frame Cul3 mRNA lacking exon 9, resulting in deletion of 57 amino acids (403-459) in CUL3. The exact role of this altered CUL3 (referred to here as CUL3- $\Delta 9$ ) in the pathogenesis of FHHt is still unclear. Several in vitro models have been proposed to explain the mechanism by which CUL3- $\Delta 9$ causes FHHt, all of which suggests that impaired CRL function leads to increased WNK4 abundance. We previously proposed that CUL3- $\Delta 9$ is an overactive form of CUL3 that inappropriately degrades KLHL3 (28). The Sigmund group reported that WT CUL3/CUL3- $\triangle 9$ heterodimers are less stable than CUL3 WT/WT homodimers, and that CUL3- $\Delta 9$ may also sequester adapters (29). Finally, the Kurz group confirmed our finding with respect to inappropriate degradation of KLHL3 but also reported that CUL3- $\Delta 9$ displays autoubiquitination, leading to its own proteasomal degradation (30). A major limitation with most of these studies has been the reliance on in vitro approaches, but the Kurz group also generated a mouse model of FHHt by targeted deletion of exon 9 of the Cul3 gene. These mice displayed an FHHt phenotype, but importantly, CUL3- $\Delta 9$ was present at extremely low levels, consistent with the observation that it autoubiquitinates in vitro. WT CUL3 protein was present at half the amount present in WT mice, suggesting it is not a target of CUL3- $\Delta$ 9. It was concluded that FHHt is most likely caused by Cul3 haploinsufficiency and that mice heterozygous for Cul3 should also display an FHHt phenotype. This has become the prevailing model for CUL3- $\Delta$ 9-mediated FHHt. Evidence against this model comes from an effort by the Uchida group to generate an FHHt mouse model carrying a splice site mutation in the C-terminus of intron 8 of Cul3, c. $1207-1 \mathrm{G}>\mathrm{A}$ (Cul3 ${ }^{\mathrm{G}[-1] \mathrm{A} /+}$ knock-in mice). Most likely as a result of differences in splicing between mouse and human, this mutation led to complete disruption of CUL3, rather than expression of CUL3- $\Delta$ 9. Mice heterozygous for $\mathrm{Cul}^{\mathrm{G}(-1) \mathrm{A} /+}$ mice did not exhibit an FHHt phenotype (31). One limitation of this model is that the splice site mutation may have produced other effects, such as expression of a hypomorphic form of CUL3 that differs functionally from CUL3- $\Delta 9$ (32).

Thus, the precise mechanism by which CUL3- $\Delta 9$ leads to FHHt is still controversial and important to unravel, since pharmacologically stimulating CUL3 activity offers a potentially attractive therapeutic approach toward development of novel antihypertensive therapies. On the contrary, if the haploinsufficiency model is proven incorrect, alternative approaches toward a CUL3-based therapy may be required (10). To directly test the hypothesis that Cul3 haploinsufficiency is sufficient to cause FHHt, we generated and compared several potentially novel mouse models. All models use the Pax8-rtTA/LC1 system to allow doxycyline-inducible excision at loxP sites specifically in renal epithelia in adult mice (33). Firstly, excision of a single endogenous floxed $\mathrm{Cul3}$ allele produces mice heterozygous for $\mathrm{Cul3}$, thus phenocopying the haploinsufficient state. The second combines the first model with a lox-STOP-lox-driven transgene to produce Cul3 heterozygotes (CUL3-Het) that also express CUL3- $\Delta 9$ (34). The final model expresses CUL3- $\Delta 9$ on a background of 2 WT Cul3 alleles. The latter 2 models test the model of dominance. Our data show that haploinsufficiency is not sufficient to cause FHHt, but introduction of CUL3- $\Delta 9$ on a haploinsufficient background leads to the disease phenotype. Introducing CUL3- $\Delta 9$ on a background of 2 WT alleles also produces features of the disease, proving that CUL3- $\Delta 9$ exerts a dominant effect. Finally, our data show that CUL3- $\Delta 9$ exerts strong dominant effects, despite its extremely low abundance, which is likely a result of rapid degradation following autoubiquitination. 


\section{Results}

CUL3-Het carried a single endogenous floxed Cul3 in addition to the inducible Cre-driver transgenes (28). CUL3-Het/ $\Delta 9$ mice additionally carried a randomly integrated transgene encoding CUL3- $\Delta 9$. The transgene included a floxed transcription blocker (STOP) downstream of the artificial CAG promoter and cDNA encoding tdTomato (a spontaneously fluorescing reporter) (34). An internal ribosome entry site (IRES) between CUL3- $\Delta 9$ and tdTomato permitted their expression separately from a single transcript. In both models, doxycycline treatment excised the floxed Cul3 allele, producing heterozygosity. In CUL3-Het/ $\triangle 9$ mice, it also removed the STOP, simultaneously causing haploinsufficiency and inducing expression of CUL3- $\Delta 9$, specifically in renal epithelial cells (Figure $1 \mathrm{~A}$ ).

We confirmed recombination and removal of the lox-STOP-lox following doxycycline administration in CUL3-Het/ $\triangle 9$ mice, but not in control mice, by PCR using genomic DNA. Pax8-rtTA-Cre-driven recombination was detected in kidney and also in liver, but it was absent from all other major organs (Figure 1B), as previously reported (33). Activation of the transgene along renal epithelia was confirmed by clear detection of tdTomato fluorescence in cortex and medulla of CUL3-Het/ $\Delta 9$ mice, with renal expression confirmed by Western blotting (Figure 1C). Western blotting showed that CUL3 abundance was significantly reduced in both CUL3-Het and CUL3-Het/D9 compared with untreated controls (Figure 1D). Although it appears that there may be a greater decrease in CUL3 in CUL3-Het/ $\triangle 9$ mice, direct comparison of the 2 strains indicates similar abundance of CUL3 (Figure 1E). While we observed the expected $\sim 50 \%$ reduction in CUL3WT in CUL3-Het/ $\Delta 9$ mice, we could not detect CUL3- $\Delta 9$. The Sigmund group was also unable to detect CUL3- $\Delta 9$ in aorta from mice expressing CUL3- $\Delta 9$ from the same transgene using a vascular smooth muscle cell (VSMC) Cre driver, but they observed a strong tdTomato signal (34). They confirmed functionality of the transgene by culturing primary aortic smooth muscle cells from CUL3- $\Delta 9$ transgenic mice and infecting them with Cre recombinase adenovirus, which resulted in low but detectable CUL3- $\Delta 9$ expression (34). Therefore, we cultured primary renal epithelial cells from CUL3-Het and CUL3-Het/ $\Delta 9$ mice and infected them with Cre adenovirus to induce transgene recombination. CUL3- $\Delta 9$ protein was detectable in infected primary cells from CUL3-Het/ $\Delta 9$ mice but not from CUL3-Het mice, with the size of CUL3- $\Delta 9$ confirmed using lysates from HEK293 cells transfected with plasmids encoding the transgene and Cre (Figure 1F). CUL3- $\Delta 9$ was expressed at extremely low levels, consistent with its ability to initiate its own degradation via autoubiquitination (30). CUL3 has been proposed to be activated by covalent linkage of an 81-amino acid protein Neural precursor cell expressed developmentally downregulated protein 8 (Nedd8), a process called neddylation that is specific to Cullins (35). Importantly, the region deleted in CUL3- $\Delta 9$ has been shown to interact with a large protein complex, the COP9 signalosome, which removes Nedd8 from Cullins (30). We found that CUL3- $\Delta 9$ displays a high degree of neddylation, consistent with its increased activity toward itself, KLHL3, and WNKs (28). To determine whether there was an increase in neddylated species in CUL3-Het/ $\Delta 9$ mice, we performed Western blotting for Nedd8. While there was no obvious difference in Nedd8 abundance between controls and CUL3-Het or CUL3-Het/ $\Delta 9$ mice, the signal obtained from kidney lysates of CUL3-Het/ $\Delta 9$ mice appeared qualitatively different and ran more as a smear. It is possible this represents neddylated CUL3- $\Delta 9$, since it runs lower than the band in control. We measured the band depth using ImageJ $(\mathrm{NIH})$, and quantification revealed it was significantly greater in CUL3-Het/ $\Delta 9$ lysates (Supplemental Figure 2; supplemental material available online with this article; https://doi.org/10.1172/jci.insight.96700DS1), suggesting the presence of a highly neddylated species, possibly CUL3- $\Delta$. We previously reported that, in transfected HeLa cells, WT CUL3 is almost exclusively localized to the nucleus, but CUL3- $\Delta 9$ mislocalizes to the cytoplasm (28). Although we did not determine if CUL3- $\Delta 9$ induces WT CUL3 mislocalization in vitro, we compared CUL3 localization in CUL3-Het and CUL3-Het/ $\Delta 9$ mice by performing immunofluorescence (IF) for CUL3. In CUL3-Het mice, CUL3 displayed strong nuclear localization along DCT1 (parvalbumin colocalization), DCT2/connecting tubule (CNT) (calbindin colocalization), and other segments (Figure 2). In contrast, in CUL3-Het/ $\Delta 9$ mice, CUL3 expression was more diffuse along parvalbumin- and calbindin-positive segments, with no strong nuclear staining, despite apparently normal nuclei (shown by DAPI staining). CUL3 staining in other segments appeared to retain nuclear localization.

We next determined the abundances of phospho-T53 NCC (pNCC) (a surrogate for NCC activity) and total NCC ( $\mathrm{tNCC}$ ). Both control and CUL3-Het mice had similar abundances of pNCC and tNCC abundance (Figure 3A), while both pNCC and tNCC, as well as pNCC/tNCC, were dramatically elevated in CUL3-Het/ $\Delta 9$ mice compared with controls (Figure $3 \mathrm{~B}$ and Supplemental Figure 3). We confirmed that the abundances of $\mathrm{pNCC}$, tNCC, and $\mathrm{pNCC} / \mathrm{tNCC}$ were higher in CUL3-Het/ $\Delta 9$ than in CUL3-Het mice by directly comparing 
A

Cul3-Het:

Cul3-Het/ $\Delta$ :
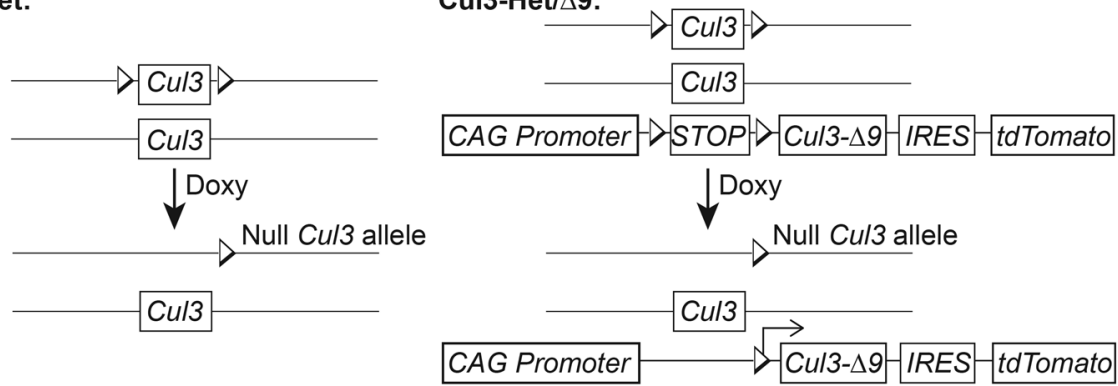

B

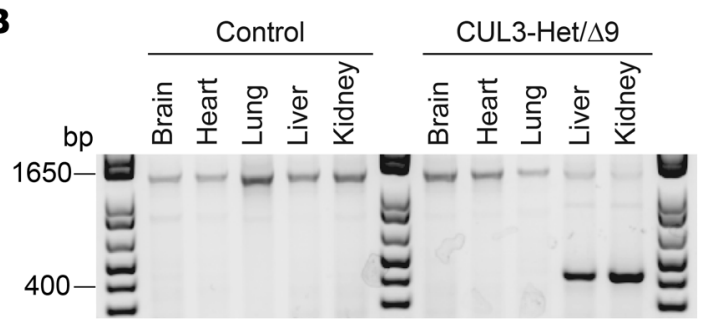

C

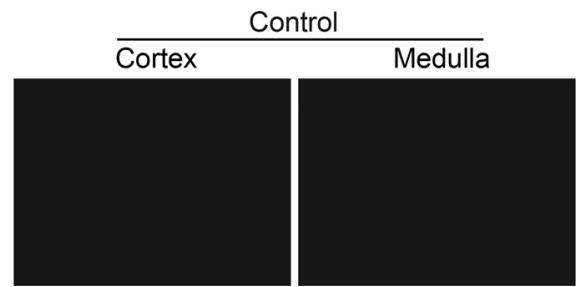

D
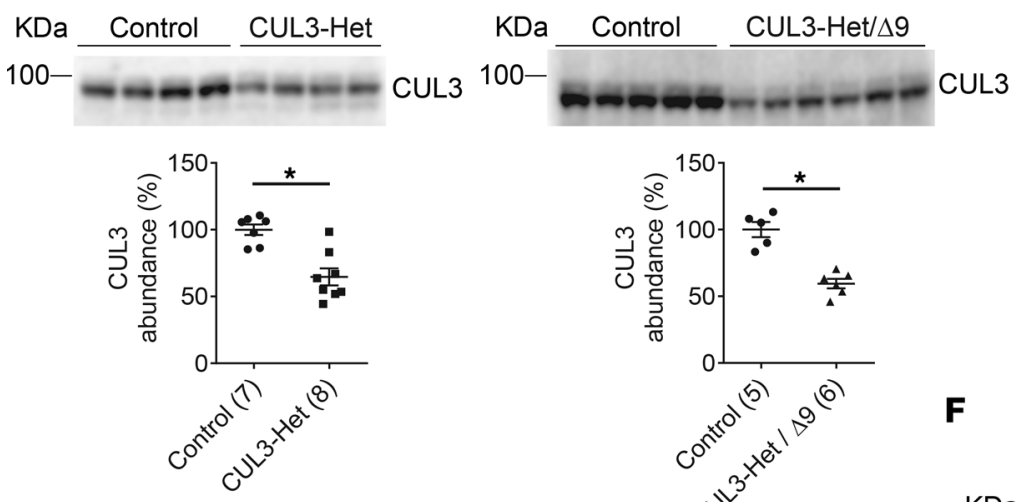

E
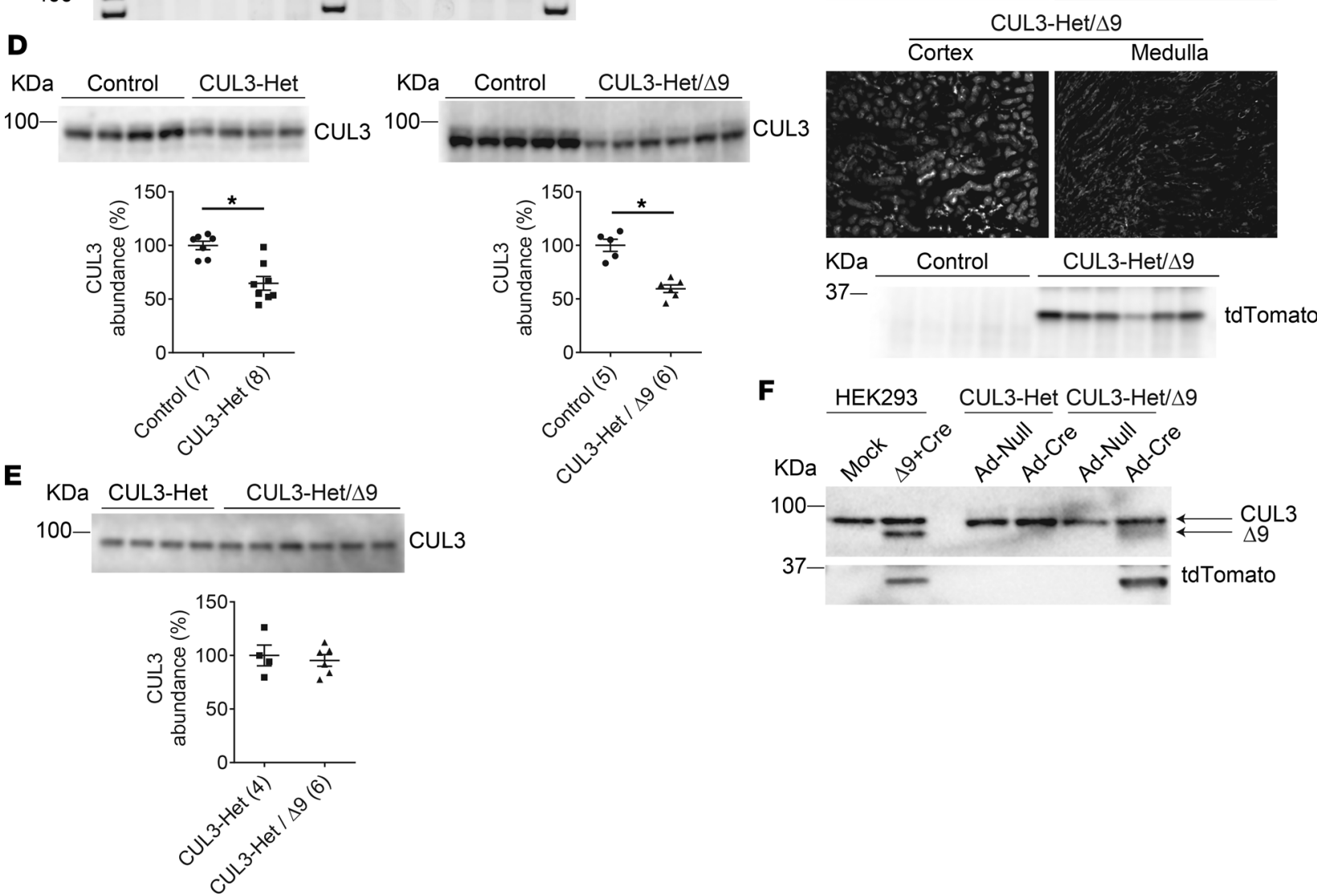

CUL3

$\mathbf{F}$

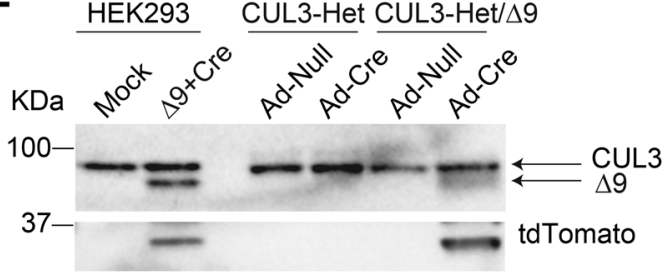

Figure 1. Generation of CUL3-Het and CUL3-Het/49 mice. (A) The Pax8-rtTA-LC1 system (present in all mice) was used for doxycycline-induced Cremediated recombination at loxP sites specifically in renal epithelia. Cul3 heterozygous mice (CUL3-Het mice, left) carried 1 WT Cul3 allele and a Cul3 allele with exons 4-7 floxed. CUL3-Het/ $\Delta 9$ mice (right) additionally carried a transgene fragment consisting of a strong synthetic promoter (CAG) promoter, a floxed transcription blocker (STOP), cDNA encoding Cul3 $\Delta 403-459$ (CUL3- $\Delta 9$ ), an internal ribosome entry site (IRES), and cDNA encoding the fluorophore $t d$ Tomato; the IRES permitted translation of CUL3- $\Delta 9$ and tdTomato separately from the same transcript. Doxycycline (Doxy, $2 \mathrm{mg} / \mathrm{ml}$ in $5 \%$ sucrose drinking water vehicle for 2 weeks) treatment induced recombination, disrupting 1 Cul3 allele in both CUL3-Het and CUL3-Het/ $\triangle 9$ mice, and - in CUL3Het $/ \Delta 9$ mice only - initiating expression of CUL3- $\Delta 9$ and tdTomato. Control mice were genetically identical to either CUL3-Het or CUL3-Het/ $\Delta 9$ mice and were given vehicle only. (B) To confirm recombination in CUL3-Het/ $\Delta 9$ mice following Doxy treatment, PCR was performed on genomic DNA from several tissues. In control mice, a 1,300 bp product, consistent with no recombination, was detected in brain, heart, lung, liver, and kidney. In CUL3-Het/ $\Delta 9$ mice, a 430 bp product derived from the recombined transgene was detected in kidney and liver, but not in brain, heart, or lung. Pax8-rtTA activity in liver has been reported previously (33). (C) tdTomato fluorescence was observed in renal cortex and medulla in CUL3-Het/ $\Delta 9$ mice, but not in controls (or CUL3-Het mice; data not shown), representative of 8 independent experiments, and Western blot of whole kidney confirmed transgene activation following recom- 
bination. (D) Western blot of whole kidney lysate showed that both CUL3-Het $\left({ }^{*} P=0.02,2\right.$-tailed unpaired $t$ test) and CUL3-Het $/ \Delta 9$ ( ${ }^{*} P=0.0001,2$-tailed unpaired $t$ test) mice had significantly reduced WT Cullin 3 (CUL3) abundance compared with controls. For quantification, densitometric values were normalized using Coomassie stained gels (see Supplemental Figure 1 for details); values in parentheses represent $n$. (E) Direct comparison of CUL3-Het and CUL3-Het/ $\Delta 9$ mice confirmed no difference in CUL3 abundance. (F) Western blot of cell lysates from primary renal epithelial cells infected with empty adenovirus (Ad-Null) or Cre adenovirus (Ad-Cre) confirmed Cre-mediated CUL3- $\Delta 9$ expression. Primary cells from uninduced CUL3-Het and CUL3-Het/ $\Delta 9$ mice were then infected with virus for 48 hours. The short infection time was not sufficient to reduce endogenous CUL3 abundance. Lysates from HEK 293 cells transfected with plasmids encoding the transgene $(\Delta 9)$ and Cre confirmed the size of CUL3- $\Delta 9$ in primary cells.

the 2 strains (Supplemental Figure 4). Higher pNCC/tNCC confirmed that the increased pNCC in CUL3-Het/ $\triangle 9$ mice was not only due to increased tNCC abundance, but also reflected an increased level of NCC phosphorylation. We next determined the abundance of WNK4, which is likely to be the major target of the CRL along the DCT $(16,23,24,26)$. Control and CUL3-Het mice had comparable WNK4 abundance (Figure 3C), while CUL3-Het/ $\Delta 9$ mice displayed higher WNK4 abundance than controls (Figure 3D). These observations suggest that WNK4 was stabilized in CUL3-Het/ $\Delta 9$ mice, leading to enhanced NCC phosphorylation; this did not occur in CUL3-Het mice, suggesting that haploinsufficency is not sufficient to cause FHHt.

FHHt due to CUL3- $\Delta 9$ causes the most severe form of the disease, with $94 \%$ of affected individuals developing hypertension by 18 years of age, and plasma $\left[\mathrm{K}^{+}\right]$averaging $7.5 \mathrm{mmol} / 1$ (4). While it has been demonstrated that CUL3- $\Delta 9$ expression can cause a primary defect in the vasculature independently of possible secondary effects of hypertension $(30,34)$, we sought to examine the possibility that increased activation of the $\mathrm{Na}^{+} \mathrm{K}^{+} 2 \mathrm{Cl}^{-}$cotransporter (NKCC2), expressed along the thick ascending limb (TAL), plays a role in CUL3- $\triangle 9$ mediated FHHt. Like NCC, NKCC2 is activated via amino-terminal phosphorylation through SPAK/OSR1 both in vitro and in vivo $(6,36-38)$, but it may also be a direct target of WNK4 (39). Importantly, CUL3 (28), KLHL3 (28), and WNK4 $(40,41)$ are all expressed along the cortical TAL. The abundances of phospho-T96/ T101NKCC2 (pNKCC2) and total NKCC2 (tNKCC2), as well as the pNKCC2/tNKCC2 ratio, were similar in control and CUL3-Het mice (Figure $4 \mathrm{~A}$ ). In contrast, CUL3-Het/ $\Delta 9$ mice displayed significantly higher abundance of $\mathrm{pNKCC} 2$ (Figure 4B), but not $\mathrm{tNKCC} 2$, reflected by a much greater $\mathrm{pNKCC} 2 / \mathrm{tNKCC} 2$ (Figure 4B), suggesting that CUL3- $\Delta 9$ exerts effects along the TAL that may contribute to FHHt.

Several in vitro and in vivo studies have reported that WNK1 and WNK4 may alter activity of ENaC (1), and in FHHt, decreased $\mathrm{Na}^{+}$flux through this channel may contribute to hyperkalemia by reducing the electrogenic drive for $\mathrm{K}^{+}$secretion. To determine whether $\mathrm{Na}^{+}$flux through $\mathrm{ENaC}$ was affected by CUL3- $\Delta$, we performed an amiloride response test - a better measurement than Western blotting or IF for $\mathrm{ENaC}$, since it directly assesses $\mathrm{Na}^{+}$flux through the channel. CUL3-Het/ $\Delta 9$ mice displayed blunted natriuretic but unchanged kaliuretic responses following an acute amiloride bolus (Figure $4 \mathrm{C}$ ).

To determine whether elevated $\mathrm{pNCC}$ in CUL3-Het/ $\Delta 9$ mice resulted in phenotypic consequences consistent with $\mathrm{FHHt}$, we measured plasma $\left[\mathrm{K}^{+}\right]$and blood pressure. CUL3-Het/ $\Delta 9$ mice displayed significantly higher plasma $\left[\mathrm{K}^{+}\right]$than control and CUL3-Het mice (Figure $5 \mathrm{~A}$ ). Administration of hydrochlorothiazide for 3 days normalized plasma $\left[\mathrm{K}^{+}\right]$in CUL3-Het/ $\Delta 9$ mice (Figure $5 \mathrm{~B}$ ). CUL3-Het/ $\Delta 9$ mice also had higher systolic blood pressure (SBP) than CUL3-Het mice (Figure 5B) and controls (Supplemental Figure 5). To further confirm that reduced abundance of CUL3 alone is not sufficient to cause an FHHt phenotype, we generated an additional mouse model in which the CUL3- $\Delta 9$ transgene was expressed in the presence of 2 WT Cul3 alleles (CUL3-WT/ $\Delta 9$ mice). CUL3-WT/ $\Delta 9$ mice and vehicle controls (CUL3-WT mice) displayed similar abundances of WT CUL3 (with CUL3- $\Delta 9$ undetectable as in CUL3-Het/ $\Delta 9$ mice) (Figure 5C), but pNCC, pNKCC2, and WNK4 abundances were all significantly higher in CUL3-WT/ $\Delta 9$ mice. However, these effects were more variable than those seen in CUL3-Het/ $\Delta 9$ mice, possibly reflecting the presence of a greater number of WT-WT CUL3 homodimers, which could overcome the effects of CUL3- $\Delta 9$ and promote WNK4 degradation. An alternative explanation is that tdTomato abundance appears to correlate with abundances of WNK4 and pNCC, so CUL3- $\Delta 9$ transgene expression may be lower in some animals. These data show that undetectable levels of CUL3- $\Delta 9$ can exert strong dominant effects that activate NCC, even when abundance of WT CUL3 is at normal levels. Perhaps expression of CUL3- $\Delta 9$ in the vasculature on a heterozygote background might increase blood pressure to a greater degree than it did on a WT background (34).

WNK4 and SPAK have been shown to localize to intracellular puncta in SPAK-KO mice (42) and following dietary $\mathrm{K}^{+}$restriction $(43,44)$. The precise identity and composition of these puncta, and their role in regulation of NCC activity, is currently unknown. Localization of WNK4 and SPAK to puncta was also observed by the Kurz group (30) but was not specifically localized with regard to nephron segment. Therefore, we performed IF to address this question. In controls and CUL3-Het mice, WNK4 signal was low in cytoplasm in cortex (Figure 

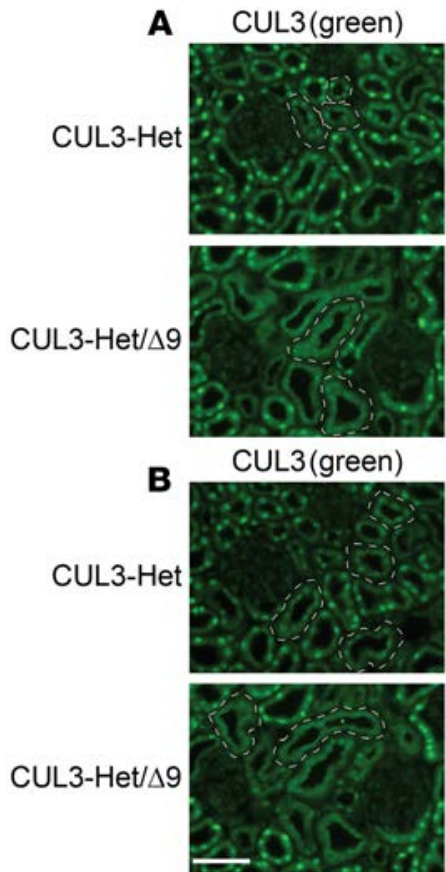
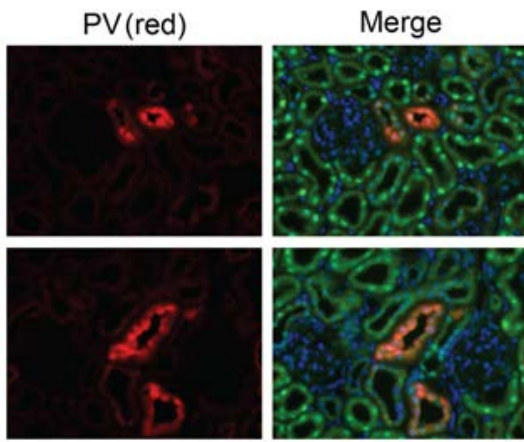

$\mathrm{CB}($ red)
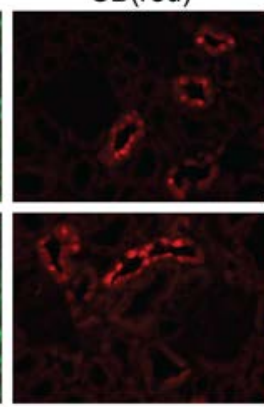

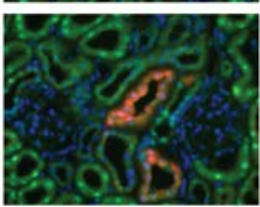

Merge

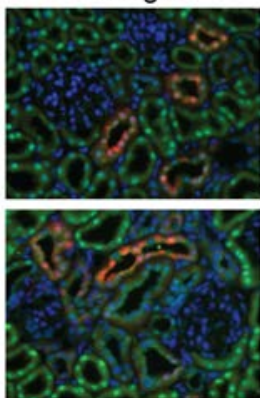

Figure 2. CUL3 distribution shifts from the nucleus to cytoplasm in CUL3-Het/ $\Delta 9$ mice in the distal nephron. Immunofluorescence for CUL3 was performed using an antibody validated in kidney-specific CUL3-KO mice. (A) In CUL3-Het mice, CUL3 displayed strong nuclear localization in most tubules, including early distal convoluted tubule (DCT1), confirmed by colocalization with parvalbumin (PV). In CUL3-Het/ $\Delta 9$ mice, CUL3 still localized to nuclei in most segments but appeared more diffuse in PV-positive segments, suggesting redistribution from the nucleus to the cytoplasm. (B) A similar redistribution was seen in tubules expressing calbindin (CB) which is also expressed along late distal convoluted tubule (DCT2), connecting tubule (CNT), and cortical collecting duct (CCD). In (A and B) PV- and CB-positive tubules are outlined on CUL3 images to assist with identification. Representatives of 2 independent experiments. Scale bar: $50 \mu \mathrm{m}$.

6A) and also low at the apical membrane in medulla (Supplemental Figure 6). In CUL3-Het mice, SPAK and OSR1 - the downstream effectors of WNK4 - were localized to the apical membrane in medulla (Supplemental Figure 6) and at the apical membrane and in small cytoplasmic puncta in cortex (Figure 6B and Supplemental Figure 7). In CUL3-Het/ $\Delta 9$ mice, WNK4, SPAK, and OSR1 were present not only in small puncta, but also in large intracellular puncta in cortex (Figure 6 and Supplemental Figure 7); however, they were not present not in medulla (Supplemental Figure 6). Colocalization with $\mathrm{tNKCC} 2$ revealed that WNK4 and SPAK puncta were absent along TAL, while other markers revealed their localization to DCT1 (parvalbumin), both DCT1 and DCT2 (NCC), and more distal segments (calbindin) (Figure 6C). Similarly, WNK1 abundance was low in CUL3-Het mice along DCT and CNT, with a signal present in small puncta; in CUL3-Het/ $\Delta 9$ mice, these puncta appeared much larger (Supplemental Figure 8). For SPAK, we performed confocal microscopy (Supplemental Figure 9A) and quantified the number and size of puncta (Supplemental Figure 9, B-D). While there were no differences in the average number of puncta per area of tubule (Supplemental Figure 9B), the puncta were significantly larger in size (Supplemental Figure 9C) in CUL3-Het/ $\Delta 9$ mice than in control and CUL3-Het mice (controls $0.16 \pm 0.01 \mu \mathrm{m}^{2}$, CUL3-Het $0.14 \pm 0.01 \mu \mathrm{m}^{2}$, CUL3-Het/ $\Delta 90.74 \pm 0.09 \mu \mathrm{m}^{2}$ ). Stratification by puncta size (cutoffs $\leq 0.6 \mu \mathrm{m}^{2}$ and $>0.6 \mu \mathrm{m}^{2}$ puncta) revealed a trend toward fewer smaller puncta per unit area of tubule and a significantly greater number of larger puncta per area of tubule in CUL3-Het/ $\Delta 9$ mice than in control and CUL3-Het mice (Supplemental Figure 9D).

\section{Discussion}

The mechanism by which CUL3- $\Delta 9$ leads to FHHt is controversial, but this is primarily related to the fact that most studies have been performed in vitro $(28-30,34)$. A constitutive deletion of exon 9 in mice suggested that FHHt is due to haploinsufficiency resulting from degradation of CUL3- $\Delta 9$ following autoubiquitination (30). Here, we directly tested this hypothesis by generating mice haploinsufficient for Cul3, expressing or not expressing CUL3- $\Delta 9$. Similar to previous CUL3- $\Delta 9$ mouse models that reported low or undetectable levels of the protein $(30,34)$, we were only able to detect CUL3- $\Delta 9$ in cultured primary cells, providing further evidence 
A
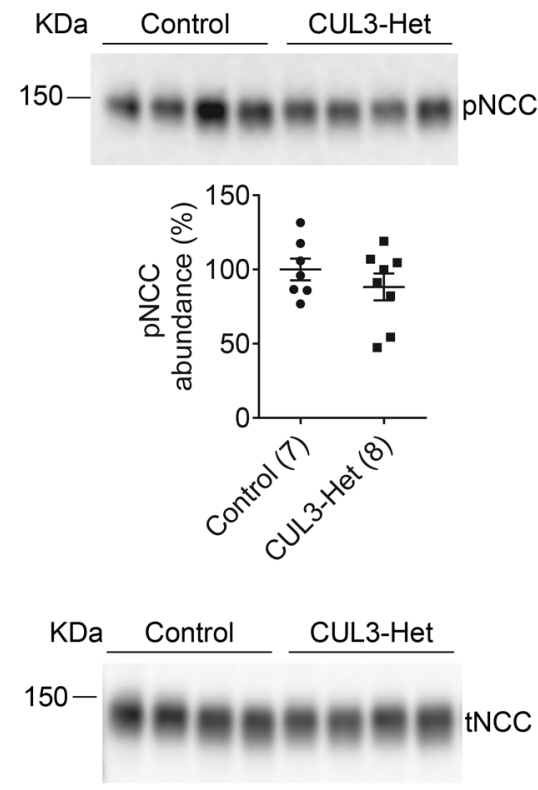

B
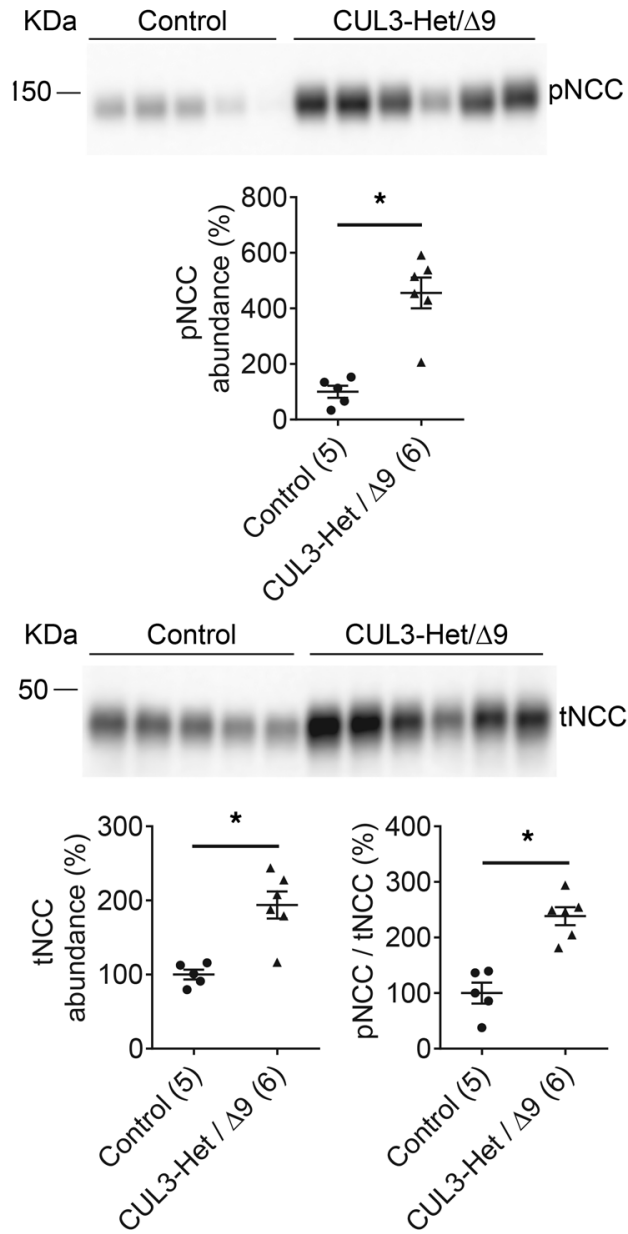

\section{C}
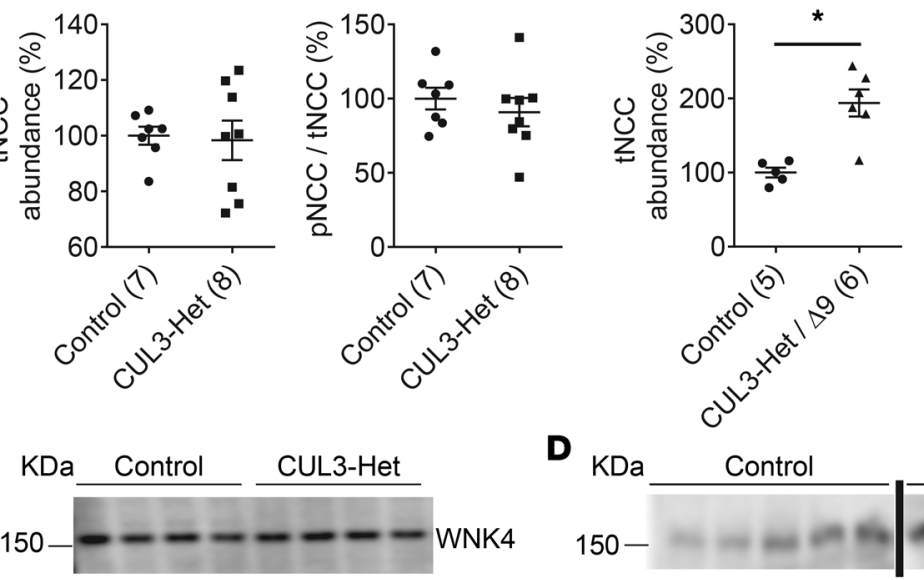

CUL3-Het/ $\Delta 9$

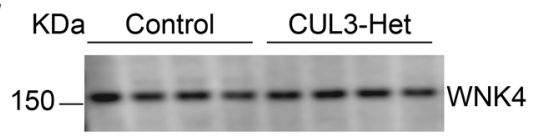

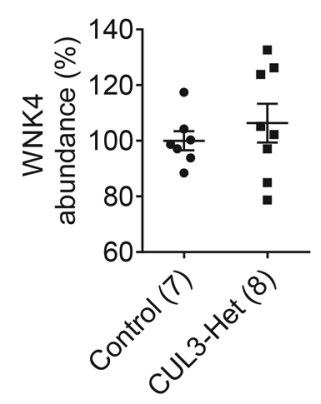

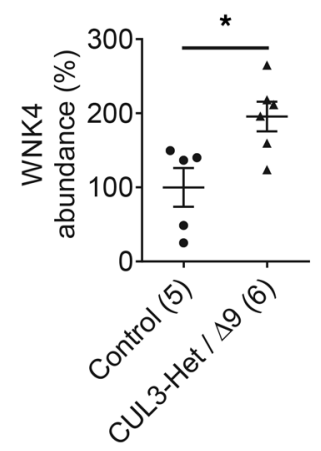

Figure 3. Cul3 heterozygosity does not increase abundances of phosphorylated $\mathrm{Na}^{+}-\mathrm{Cl}^{-}$cotransporter (NCC) and With-No-Lysine [K] Kinase 4 (WNK4), but requires additional effects of CUL3- $\Delta$ 9. (A) Western blotting of whole kidney lysate showed that abundances of NCC phosphorylated at threonine 53 (pNCC), total NCC (tNCC), and the ratio $\mathrm{pNCC} / \mathrm{tNCC}$ did not differ between control and CUL3-Het mice. (B) Compared with controls, both pNCC $\left(^{*} P=0.0003\right.$, 2-tailed unpaired $t$ test) and $\mathrm{tNCC}\left({ }^{*} P=0.02,2\right.$-tailed unpaired $t$ test) were significantly greater in CUL3-Het/ $\Delta 9$ mice. CUL3-Het/ $\Delta 9$ mice also had significantly higher pNCC/tNCC $\left({ }^{*} P=0.0003\right.$, 2-tailed unpaired $t$ test) than control mice, suggesting increased NCC phosphorylation was not just due to similar WNK4 abundances. (C) WNK4 abundance did not differ between controls and CUL3-Het mice. (D) WNK4 abundance was higher in CUL3-Het/ $\Delta 9$ mice than in control mice $\left({ }^{*} P=0.01\right.$, 2-tailed unpaired $t$ test). For quantification, densitometric values were normalized using Coomassie stained gels (see Supplemental Figure 1 for details); values in parentheses indicate $n$. 
A
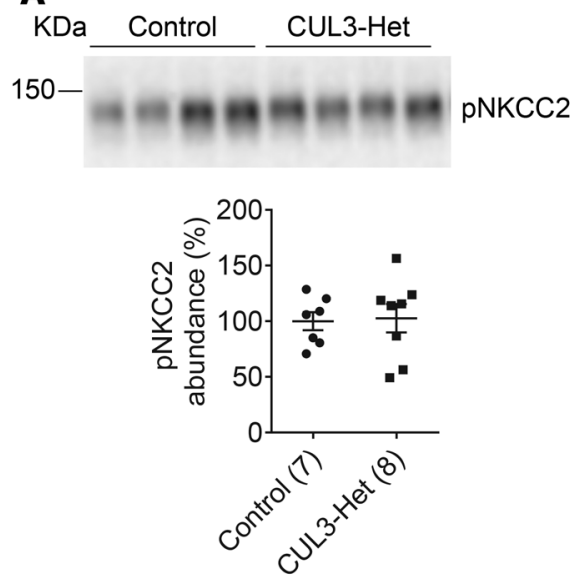

B

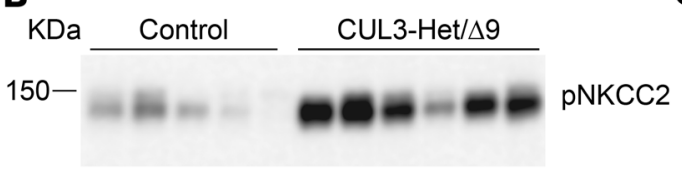

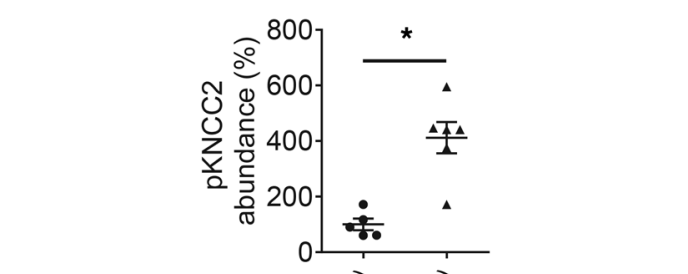
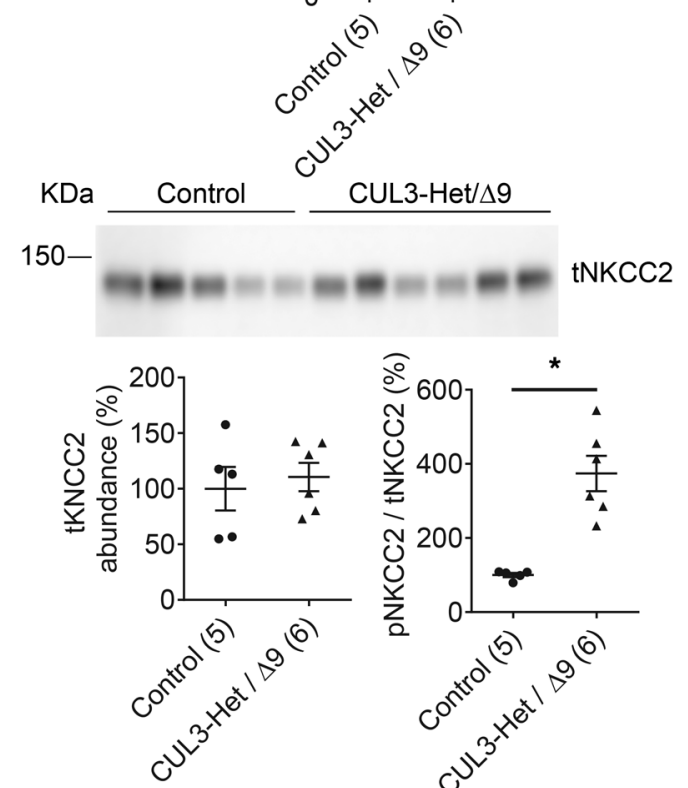

C
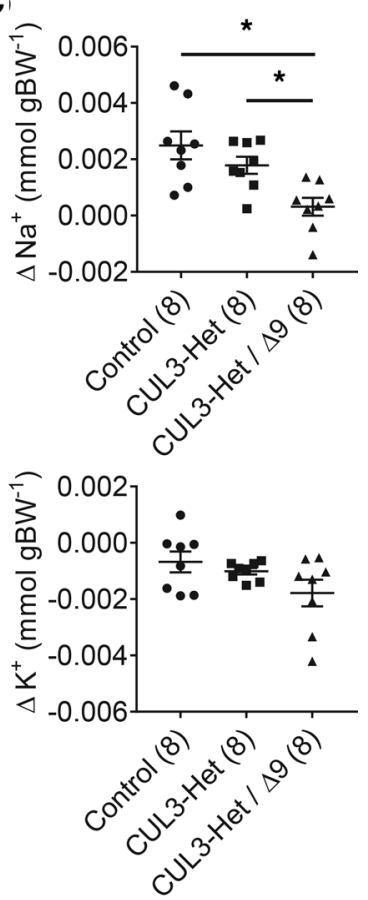

Figure 4. Evidence for increased $\mathrm{Na}^{+}-\mathrm{K}^{+}-2 \mathrm{Cl}^{-}$cotransporter (NKCC2) activation and reduced $\mathrm{Na}^{+}$flux through the epithelial sodium channel (ENaC) in CUL3-49-mediated FHHt. (A) Western blotting of whole kidney lysate showed that control CUL3-Het mice had similar abundances of NKCC2 phosphorylated threonines 96 and 101 (pNKCC2) and total NKCC2 (tNKCC2), and a similar pNKCC2/tNKCC2 ratio. (B) CUL3-Het/D9 mice displayed significantly increased abundance of pNKCC2 than controls ( ${ }^{*} P=0.0009$, 2-tailed unpaired $t$ test). tNKCC2 did not differ, but pNKCC2/tNKCC2 was significantly higher in CUL3-Het $/ \Delta 9$ mice compared with controls $\left({ }^{*} P=0.0005\right.$, 2-tailed unpaired $t$ test). For quantification, densitometric values were normalized using Coomassie stained gels (see Supplemental Figure 1 for details). (C) To determine whether Na+ flux through ENaC was altered in CUL3-Het/ $\Delta 9$ mice, an amiloride response test was performed. Vehicle ( $0.09 \%$ saline) was injected and urine collected for 6 hours; the next day, amiloride ( $40 \mu \mathrm{g} 25 \mathrm{~g}^{-1}$ body weight) was injected followed by 6 -hour urine collection. The difference in $\mathrm{Na}^{+}$or $\mathrm{K}^{+}$excretion between vehicle and amiloride injection was then calculated. Compared with controls and CUL3-Het mice, CUL3-Het/ $\Delta 9$ mice displayed blunted natriuretic responses to amiloride. ${ }^{*} P<0.05$ for control versus CUL3Het/ $\Delta 9$ and for CUL3-Het versus CUL3-Het/ $\Delta 9$. One-way ANOVA, Tukey's multiple comparison test; values in parentheses indicate $n$.

that the autoubiquitination observed in vitro occurs in vivo. Importantly, the antibody we used readily detects CUL3- $\Delta 9$ in vitro (34), indicating that the inability to detect CUL3- $\Delta 9$ is not due to loss of antibody affinity. Our main conclusion is that, while CUL3- $\Delta 9$ induces haploinsufficiency by initiating its own degradation, haploinsufficiency itself is not sufficient to induce an FHHt phenotype. Introduction of CUL3- $\Delta 9$ on a haploinsufficient background elicited the FHHt phenotype, indicating CUL3- $\Delta 9$ exerts dominant effects. These dominant effects are very strong, since they occur despite very low CUL3- $\Delta 9$ abundance due to its rapid degradation. The observation that NCC is also activated following introduction of CUL3- $\Delta 9$ on a WT background, where levels of endogenous CUL3 are normal, strengthens these conclusions. Compared with control or CUL3-Het mice, CUL3-Het/ $\triangle 9$ mice display a phenotype consistent with FHHt, including increased abundances of WNK4, WNK1 and consequently pNCC, higher plasma $\left[\mathrm{K}^{+}\right]$, and higher blood pressure . It is perhaps not surprising that haploinsufficiency does not cause FHHt, since it would be predicted that mutations in Cul3 in regions that prevent interactions with KLHL3 or the RING ligase would also cause the disease; however, all cases of FHHtcausing Cul3 mutations identified specifically generate a form of CUL3 lacking a specific region involved in CUL3 inactivation (30), consistent with altered function, not loss of function. 
A
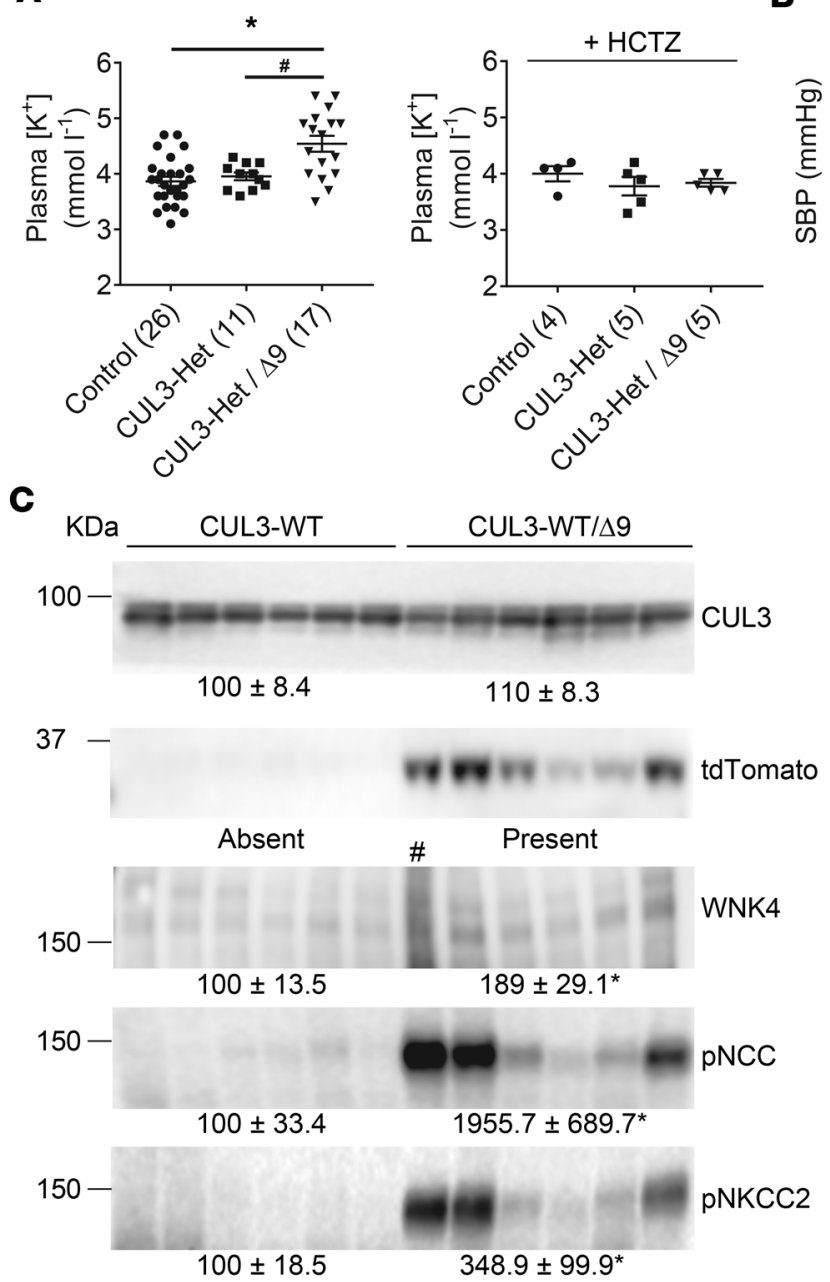

WNK4
B

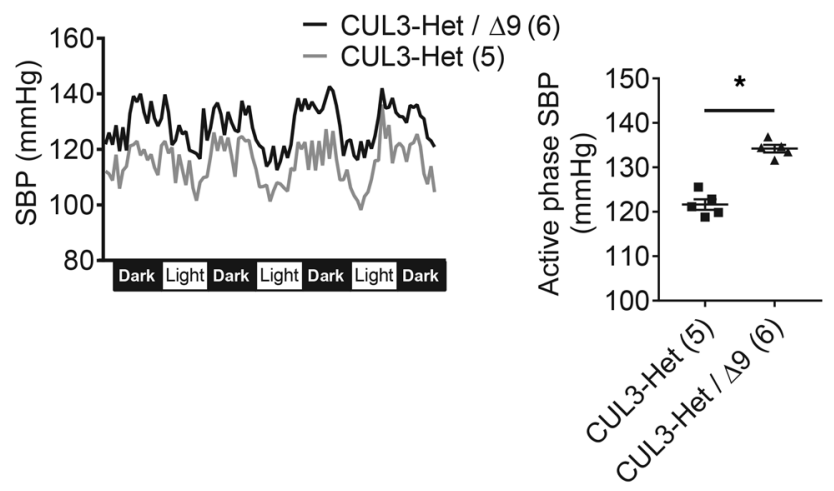

Figure 5. CUL3-Het/ $\Delta 9$ mice display increased plasma [K+] and blood pressure, and expression of CUL3- $\Delta 9$ on a WT background also activates $\mathrm{Na}^{+}-\mathrm{Cl}^{-}$ cotransporter (NCC). (A) Plasma [K+] did not differ between control and CUL3-Het mice, but it was significantly higher in CUL3-Het/ $\Delta 9$ mice (adjusted $P$ values, ${ }^{*} P<0.0001$ versus control, and ${ }^{\#} P=0.004$ versus CUL3-Het. One-way ANOVA, Tukey's multiple comparison test). Panel to right shows normalization of plasma $\left[\mathrm{K}^{+}\right]$in CUL3-Het $/ \Delta 9$ mice by administration of hydrochlorothiazide (HCTZ) for 3 days. (B) Radiotelemetric blood pressure measurement revealed higher systolic blood pressure (SBP) in CUL3-Het/ $\Delta 9$ compared with CUL3-Het mice on a normal ( $0.49 \%$ NaCl) diet. Left, trace showing 1 hour average values; right, mean of the hourly averages over 4 dark periods, \pm SEM ( ${ }^{*} P<0.0001$, 2-tailed unpaired $t$ test). (C) Western blotting of whole kidney lysate showed that, compared with WT (CUL3-WT) mice, WT mice expressing CUL3- $\Delta 9$ (CUL3-WT/ $\Delta 9$ ) displayed significantly higher abundances of WithNo-Lysine [K] Kinase (WNK4) $\left({ }^{*} P=0.01\right.$, lane marked \# was not used in analysis), phosphorylated NCC (pNCC) $\left({ }^{*} P=0.02\right)$, and phosphorylated $\mathrm{Na}^{+}-\mathrm{K}^{+}-2 \mathrm{Cl}^{-}$ cotransporter (pNKCC2) ( $\left.{ }^{*} P=0.03\right)$. Note correlation of tdTomato abundance, which may reflect CUL3- $\Delta 9$ expression, with abundances of WNK4, pNCC, and total NCC (tNCC). Densitometric values were normalized using Coomassie stained gels (see Supplemental Figure 1 for details). Values in parentheses indicate $n$; statistical tests in $\mathbf{B}$ and $\mathbf{C}$ are 2-tailed unpaired $t$ tests.

Our results provide mechanistic insight into the pathophysiological mechanisms, leading to the more severe FHHt caused by CUL3- $\Delta$ 9. They show that kidney-specific expression of CUL3- $\Delta 9$ is sufficient to cause elevations in plasma $\left[\mathrm{K}^{+}\right]$and blood pressure. Similar to an inducible mouse model for WNK4mediated FHHt (45), the phenotype was induced in adult mice, suggesting that FHHt is not simply due to developmental effects. A role for NKCC2 in blood pressure regulation is well-established, with mutations in SLC12a2 (which encodes NKCC2) causing the hypotensive disorder Type I Bartter syndrome, and use of the NKCC2 blocker furosemide as an antihypertensive. However, a possible contribution of increased NKCC2 activation to FHHt has not been reported. We found a significant increase in the abundance of $\mathrm{pNKCC} 2$ in CUL3-Het/ $\Delta 9$ mice, which may contribute to the more severe disease caused by CUL3- $\Delta$ 9. Since both NCC and NKCC2 might be activated, effects of NKCC2 blockade with furosemide may be 


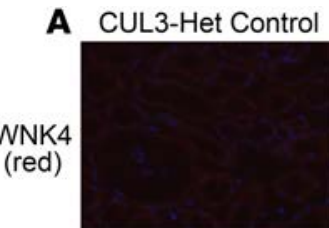

CUL3-Het

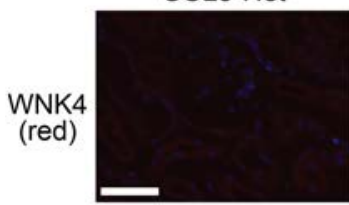

C CUL3-Het/ $\triangle 9$ :

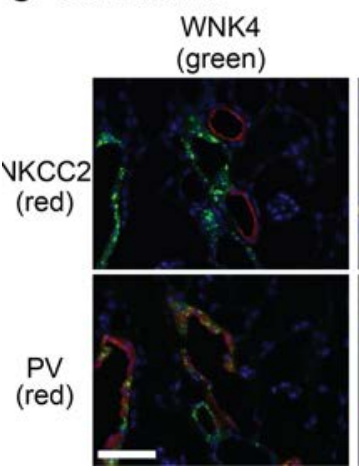

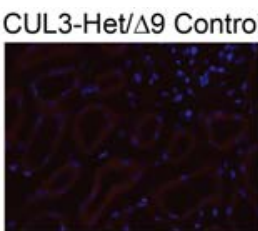

CUL3-Het/ $\Delta 9$

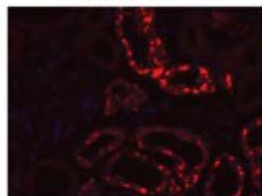

SPAK (green)

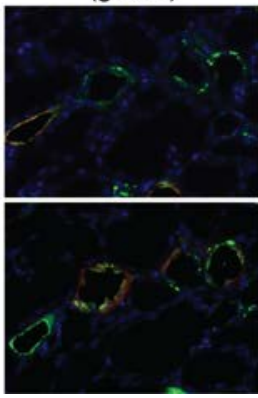

B

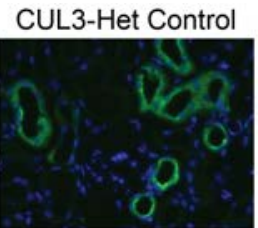

CUL3-Het
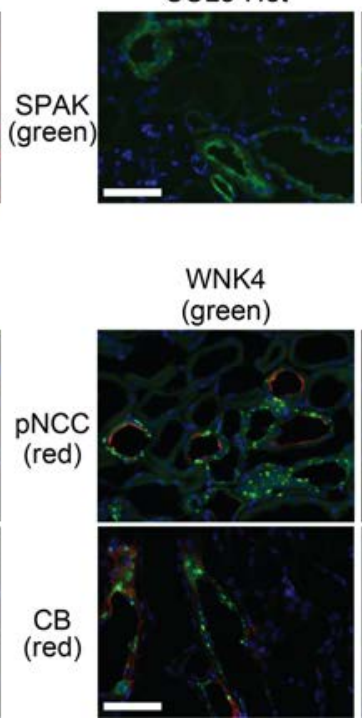

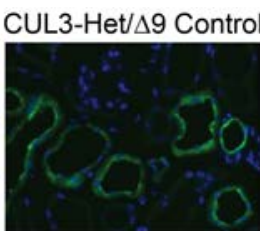

CUL3-Het/ $\Delta 9$
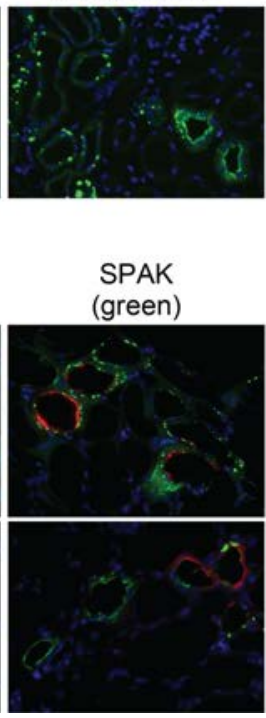

Figure 6. With-No-Lysine [K] Kinase (WNK4) and Sterile 20 (STE20)/SPS-1-related proline/alanine-rich kinase (SPAK) localize to puncta in CUL3-Het/ $\Delta 9$ mice, but not in $\mathrm{Na}^{+}-\mathrm{K}^{+}-2 \mathrm{Cl}^{-}$cotransporter-positive (NKCC2-positive) segments. (A) Immunoflourescence for WNK4 revealed weak signal in cortex in control and CUL3-Het mice. In CUL3-Het/ $\Delta 9$ mice, a strong WNK4 signal (red) was observed in large cytoplasmic puncta. (B) Immunofluorescence for SPAK (green) showed its localization at the apical membrane and in small cytoplasmic puncta in cortical segments in control and CUL3-Het mice. In CUL3-Het/ $\Delta 9$ mice, the majority was localized in large cytoplasmic puncta. (C) Colocalization studies in sections from CUL3-Het/ $\triangle 9$ mice showed that WNK4 and SPAK puncta did not colocalize with NKCC2 along the cortical thick ascending limb (cTAL). Colocalization with parvalbumin (PV) showed WNK4 and SPAK puncta were present along early distal convoluted tubule (DCT), DCT1. Colocalization with phosphorylated $\mathrm{Na}^{+}-\mathrm{Cl}^{-}$cotransporter (pNCC) showed that WNK4 and SPAK puncta were present along both DCT1 and late DCT (DCT2), since no pNCC-positive tubules lacking puncta were observed. However, some NCC-negative segments contained puncta. While calbindin (CB) is also expressed along DCT2, colocalization of puncta with CB but not pNCC indicates expression along connecting tubule (CNT) and/or cortical collecting duct (CCD). Representative of 8 independent experiments. In A-C, scale bars: $50 \mu \mathrm{m}$.

confounded, so future studies involving sequential blockade of both transporters, or generation of mice with TAL expression of CUL3- $\Delta 9$, are required to address this possibility. Furthermore, while we found that renal epithelial CUL3- $\Delta 9$ expression is sufficient to cause an FHHt phenotype, direct effects in vascular smooth muscle are also likely to contribute to the early-onset hypertension seen in patients (34).

With regard to the etiology of hyperkalemia in $\mathrm{FHHt}$, the dogma has been that increased $\mathrm{Na}^{+}$reabsorption along the DCT by NCC reduces delivery of $\mathrm{Na}^{+}$to the CNT and cortical CD (CCD), where electrogenic $\mathrm{Na}^{+}$reabsorption through $\mathrm{ENaC}$ normally provides the drive for $\mathrm{K}^{+}$secretion; this leads to hyperkalemia. Recently, evidence has emerged that challenges this mechanism. A study in mice (46) found that administration of the NCC blocker hydrochlorothiazide to WT mice did not increase kaliuresis acutely. The Welling group recently generated a mouse model with constitutive activation of SPAK specifically along the DCT1 (47), which exclusively expresses NCC, in contrast to the DCT2, which - in common with the connecting tubule (CNT) - also expresses the ENaC and ROMK (48). These mice displayed remodeling of the CNT, which was attributed solely to increased NCC activation. Reduced total and apical membrane expression of $\mathrm{ENaC}$ and $\mathrm{ROMK}$ observed was proposed to contribute to the hyperkalemia observed in FHHt by reducing $\mathrm{K}^{+}$-secreting capacity. Importantly, hydrochlorothiazide normalized these structural changes in parallel with plasma $\left[\mathrm{K}^{+}\right]$normalization but more slowly than changes in $\mathrm{Na}^{+}$excretion. We observed reduced natriuresis in response to acute amiloride injection in CUL3-Het/ $\Delta 9$ mice, but kaliuresis was not different. This effect on $\mathrm{ENaC}$ flux may not reflect a remodeling effect but may simply be 


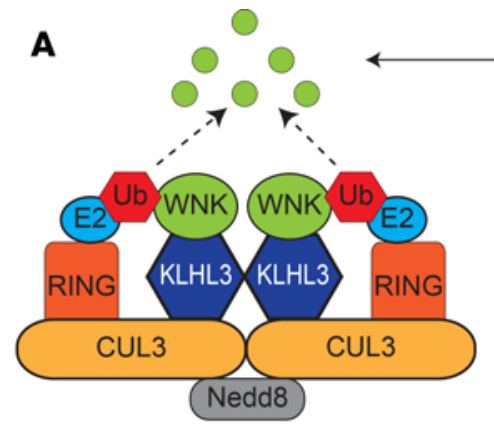

Proteasomal degradation of WNK

B

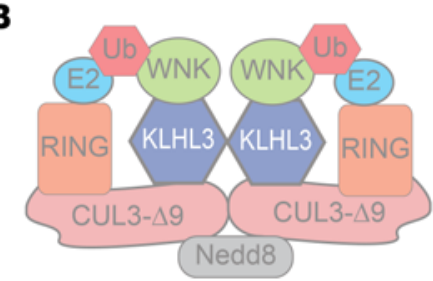

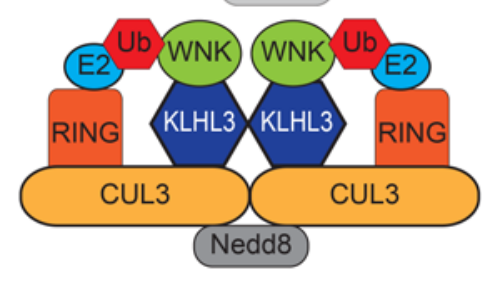

Haploinsufficiency

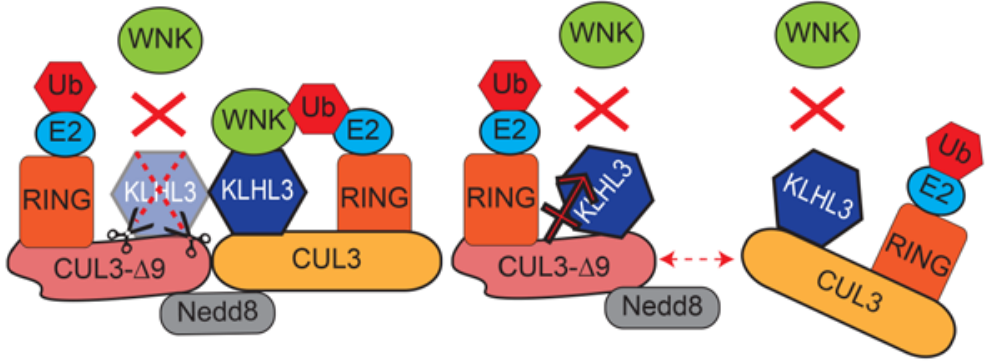

KLHL3 degradation

Sequestration by CUL3- $\Delta 9$

Figure 7. Model of normal Cullin RING Ligase (CRL) function and proposed mechanisms of CUL3- $\Delta$ 9-mediated familial hyperkalemic hypertension (FHHt). (A) The active WT CRL is a complex composed of 2 Cullin 3-containing (CUL3-containing) complexes. CUL3 acts as a scaffold for the complex. One CUL3 monomer is covalently linked to Neural precursor cell expressed developmentally downregulated protein 8 (Nedd8), which may facilitate dimerization with a non-Nedd8-conjugated CUL3 monomer and also activates the CRL (35). Each CUL3 monomer can interact with many different substrate adaptors, but Kelch-like 3 (KLHL3 is shown here since it is relevant to FHHt. WNK kinases interact with KLHL3, which brings them close to the RING E2 ubiquitin ligase that covalently attaches ubiquitin (Ub) to With-No-Lysine [K] Kinases (WNKs), targeting them for proteasomal degradation. (B) Three mechanisms have been proposed to explain how CUL3- $\Delta 9$ causes FHHt. In the haploinsufficiency model, CUL3- $\Delta 9$ triggers degradation of itself, but not of WT CUL3, by the proteasome (30). Fewer functional CRLs are available to degrade WNKs, which accumulate, leading to inappropriate $\mathrm{Na}^{+}$-Cl' ${ }^{-}$cotransporter (NCC) phosphorylation and activation by Sterile 20 (STE20)/SPS-1-related proline/alanine-rich kinase (SPAK)/oxidative stress-response kinase-1 (OSR1). Our data do not support this model. In the KLHL3 degradation model, CUL3- $\Delta 9$ inappropriately degrades KLHL3, decreasing the number of active CRLs and causing accumulation of WNKs (28). Since KLHL3 is highly expressed along the distal convoluted tubule, the site of NCC expression, this leads to FHHt, which is primarily a disease of NCC dysregulation. This model assumes that CUL3- $\Delta 9$ exerts most of its effects on KLHL3 and not on other CUL3 adaptors. Western blotting (30) and immunofluorescence for KLHL3 (Supplemental Figure 10) in CUL3- $\Delta 9$-expressing mouse models do not support this model. In the sequestration model, CUL3/CUL3- $\Delta 9$ heterodimers are less stable than CUL3/CUL3 homodimers, and CUL3- $\Delta 9$ may also sequester adapters (29), leading to a lower number of active CRLs and resulting in WNK accumulation. Our data do not refute this model, but more extensive disruption of CRL activity might be expected to cause multiple defects, not just FHHt.

due to limitation of $\mathrm{Na}^{+}$conductance arising from reduced $\mathrm{Na}^{+}$delivery, since $\mathrm{NCC}$ and $\mathrm{NKCC} 2$ are more active. It may also be due to direct effects of increased WNK abundance on ENaC activity, which have been described for WNK1 and WNK4, and could contribute to hyperkalemia by inhibiting ROMK activity (reviewed in ref. 1). Expression of CUL3 (28), KLHL3 (28), WNK1 (49), and WNK4 (40) along the CNT and CCD may support a role for direct effects in these segments.

The persistence of an antikaliuretic effect of amiloride in CUL3-Het/ $\Delta 9$ mice despite a blunted natriuretic response was surprising, given the standard view that $\mathrm{Na}^{+}$flux through $\mathrm{ENaC}$ is closely linked to $\mathrm{K}^{+}$secretion along the CNT/CCD. Mouse models of FHHt do not display the high levels of plasma $\left[\mathrm{K}^{+}\right]$ seen in humans, suggesting additional compensatory factors are at play in mice, which may confound the effects of amiloride. Uncoupling of $\mathrm{K}^{+}$secretion from $\mathrm{ENaC}$-mediated $\mathrm{Na}^{+}$reabsorption in response to amiloride is not without precedent. The Sansom group reported that, in BK- $\beta 4-\mathrm{KO}$ mice, effects on natriuresis were similar to those in WT mice, but the antikaliuretic effect was blunted (50). They proposed a role for NDCBE1 and NKCC1 in intercalated cells, and in CUL3-Het/ $\Delta 9$ mice, alterations in these and also in $\mathrm{BK}$-mediated $\mathrm{K}^{+}$secretion may play a role. Another possibility is that, as seen with chronic hydrochlo- 
rothiazide in the Welling model (47), chronic remodeling effects of amiloride may be required to diminish antikaliuresis in CUL3-Het/ $\Delta 9$ mice (note that, while blunted, amiloride still exerts a natriuretic effect in these mice). The precise mechanisms involved are beyond the scope of this study but will be the subject of future studies. Studies in isolated tubules may be particularly informative, and careful stereological analysis of the CNT and CD will be required to determine whether distal remodeling occurs in CUL3-Het/ $\Delta 9$ mice.

Emerging evidence supports a role for the DCT as the key renal sensor of plasma $\left[\mathrm{K}^{+}\right](44)$, with increased NCC activity as plasma $\left[\mathrm{K}^{+}\right]$drops serving to reduce kaliuresis, and vice versa (see above for discussion of the $\mathrm{Na}^{+}$delivery versus remodeling mechanisms of kaliuresis) (47). Redistribution of WNK1, WNK4, and SPAK to larger intracellular puncta has been reported in several mouse models with lower plasma $\left[\mathrm{K}^{+}\right]$, including SPAK KOs $(42,51,52)$, and mice fed low $\mathrm{K}^{+}$diets $(43,44,51)$. Whether puncta form when mice are placed on a high $\left[\mathrm{K}^{+}\right]$diet has not yet been reported, but they were observed in mice with targeted deletion of Cul3 exon 9, which have elevated plasma $\left[\mathrm{K}^{+}\right](30)$. We also observed large puncta in CUL3-Het/ $\triangle 9$ mice and found that they are present in DCT and more distally, but they are completely absent from TAL. These puncta, whose precise identities are unknown, may reflect the assembly of complexes that modulate activity of pathways leading to NCC activation. In CUL3-Het/ $\Delta 9$ mice, the very large puncta may represent dysregulated signaling complexes and, hence, contribute to FHHt. Alternatively, they may represent aggresomes that form due to a failure to clear accumulated WNK4 and WNK1, but the Kurz group reported they do not colocalize with the lysosomal marker LAMP1 or form ubiquitincontaining aggregates (30). Further characterization of these structures will be required to identify them and determine whether they are distinct to those seen following Spak disruption or dietary $\mathrm{K}^{+}$restriction. Our observation that these puncta were absent from TAL, despite increased WNK4 abundance, suggests differences between the effects of CUL3- $\Delta 9$ in TAL and DCT. This may reflect the key role proposed for the DCT in sensing and initiating a response to changes in plasma $\left[\mathrm{K}^{+}\right]$.

Several models have been proposed to explain the mechanism by which CUL3- $\Delta 9$ causes FHHt (Figure 7). Here, we set out to test the hypothesis that Cul3 haploinsufficiency causes FHHt. Our data strongly suggest this hypothesis is incorrect. Since haploinsufficiency is not sufficient to cause an FHHt phenotype, CUL3- $\Delta 9$ must exert dominant effects. We previously proposed that CUL3- $\Delta 9$ may cause FHHt by inappropriately degrading KLHL3, which is highly enriched along the DCT (28), but a CUL3- $\Delta 9$ model with constitutive exon 9 deletion at 1 Cul3 allele displayed no difference in KLHL3 abundance compared with WT mice (30). However, the antibody used was not validated using KLHL3-KO mice, and may also detect KLHL2 (mouse KLHL2 and KLHL3 have $87.4 \%$ similarity). We also found no difference in KLHL3 abundance using an antibody that binds both KLHL2 and KLHL3 by Western blotting (Supplemental Figure 10). Attempts to use an antibody recently shown to be specific using kidney lysates from KLHL3-KO mice (27) have thus far been unsuccessful (data not shown), but we detected no differences in KLHL3 abundance along DCT1 and DCT2/CNT by IF using this antibody (Supplemental Figure 11). Another model for CUL3- $\Delta 9$-mediated FHHt proposes that CUL3- $\Delta 9$ exerts dominant-negative effects, disrupting WT CUL3 homodimers, and possibly also causing sequestration of substrate adaptors, thus preventing substrate ubiquitination (29). Combined with the reduction of abundance of WT CUL3, WT CUL3 homodimer disruption and adapter sequestration would also be expected to further reduce activity of the WT CRL. However, floxing of the Cul3 allele alone reduces CUL3 expression by about $15 \%$, and while $\mathrm{Cul3}^{+/-}$mice are viable, global constitutive $\mathrm{Cul} 3^{\text {flox/- }}$ mice, which presumably have CRL activity lower than 50\%, are not (53). With the caveat that these data are from mice, the combination of haploinsufficiency and sequestration might also be lethal in humans rather than a cause of FHHt. Therefore, dominant-negative effects of CUL3- $\Delta 9$ are less likely to cause FHHt. We performed IF for CUL3 and found that it appears diffusely localized in some segments in mice expressing CUL3- $\Delta 9$, in contrast to the strongly nuclear localization observed in CUL3-Het mice along all segments. Interestingly, this appeared to be restricted to parvalbumin- and calbindin-positive segments. We speculate that CUL3- $\Delta 9$ may cause mislocalization of WT CUL3, specifically along the distal nephron. We previously reported mislocalization of CUL3- $\Delta 9$ itself in transfected cells (28) but did not examine effects on WT CUL3 distribution. Further studies are required to confirm this effect both in vitro and in vivo and to determine the mechanism by which this segment-specific effect might occur. One possibility is that it involves interactions with KLHL3. Since CUL3- $\Delta 9$ is degraded, it is unlikely to be present at stoichiometric levels with WT CUL3, so formation of WT/ $\triangle 9$ CUL3 heterodimers is unlikely to be a mechanism of WT CUL3 mislocalization. Additional gain of function may also be involved. 
A better understanding of how CUL3 regulates renal ion transport in both normal and disease conditions is essential before it can be targeted rationally for antihypertensive treatment. Notably, we previously reported that kidney-specific Cul3 disruption has affects in multiple segments of the nephron and causes extensive renal damage (28). Further work is required to determine the entire spectrum of CUL3 and CUL3- $\Delta 9$ functions in the kidney, but here, we show that in the etiology of FHHt, CUL3- $\Delta 9$ has potent dominant effects that go beyond simply initiating its own degradation.

\section{Methods}

Animals. The Pax8-rtTA/LC1 system was used to generate inducible renal epithelia-specific mouse models. LoxpSTOP-Loxp-Cul3-49-IRES-tdTomato transgenic mice (34) were crossed with Cul3 ${ }^{\text {flox/flox}}$-Pax8/LC1 mice (28), to

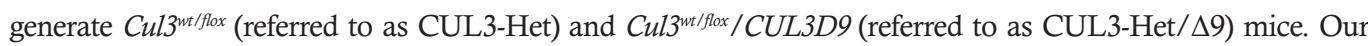
breeding scheme to generate CUL3-Het/ $\Delta 9$ mice also generated CUL3-Het mice; thus, both CUL3-Het and CUL3-Het/ $\Delta 9$ mice were littermates. CUL3-WT/ $\Delta 9$ mice, which express CUL3- $\Delta 9$ on a background of 2 WT Cul3 alleles (Cul3 $\left.{ }^{w t / w t} / C U L 3-49\right)$ following doxycycline administration, were generated by crossing Loxp-STOPLoxp-Cul3-49-IRES-tdTomato transgenic mice with Pax8-rtTA/LC1-Cul3 ${ }^{\text {wt } / w t}$ mice. All strains also had Pax8-rtTA/ $L C 1$ to permit inducible recombination in renal epithelial cells. All mice were a mixed C57B1/6J;sv129 background. Administration of $2 \mathrm{mg} / \mathrm{ml}$ doxycycline in 5\% sucrose (both Thermo Fisher Scientific) in drinking water for 2 weeks was performed to induce recombination at floxed sites. In both strains, this led to disruption of 1 endogenous $\mathrm{Cul3}$ allele and induction of transcription of mRNA encoding CUL3- $\Delta 9$ and tdTomato in those mice also carrying the Cul3D 9 transgene. Control mice given 5\% sucrose drinking water were phenotypically equivalent to WT mice and were littermates of those receiving doxycyline. Male and female mice were used for all studies, except for blood pressure measurement, which used only males, and IF, which used only females.

PCR genotyping. Genomic DNA extracts were prepared from tail snips by heating overnight at $55^{\circ} \mathrm{C}$ in $300 \mu$ digestion solution containing $5 \mathrm{mM}$ EDTA (Thermo Fisher Scientific), $200 \mathrm{mM} \mathrm{NaCl}$ (Thermo Fisher Scientific), $100 \mathrm{mM}$ Tris (pH 8.0; MilliporeSigma), 0.2\% SDS (MilliporeSigma), and $0.4 \mathrm{mg} \mathrm{ml}^{-1}$ proteinase $\mathrm{K}$ (Viagen Biotech), followed by ethanol precipitation. The following primers were used: Pax8 forward, 5'-CCATGTCTAGACTGGACAAGA-3'; Pax8 reverse, 5'-CAGAAAGTCTTGCCATGACT-3'; CRE forward, 5'-TTTCCCGCAGAACCTGAACCTGAAGAT-3'; CRE reverse, 5'-TCACCGGCATCAACGTTTTCTT-3'; Cul3 flox forward, 5'-CAGGTTGTATTTTAACTGCTTAAATGTCAAAACCT-3'; $\mathrm{Cul}^{\text {flox }}$ reverse, 5'-TTTGTCTGGACCAAATATGGCAGCCAAAACC-3'; Cul3- $\Delta 9$ forward, 5'-GGCGCGATTCTTACCAAGTCC-3'; and Cul3- $\Delta 9$ reverse, 5'-GCGCATGAACTCTTTGATGACTT-3'. To confirm recombination of the Loxp-STOP-Loxp from the CUL3D9 transgene, the following primers were used: $\triangle$ 9recombinant forward, 5'-CCTCTGCTAACCATGTTCATGCCTTCTTC-3', and $\triangle 9$ recombinant reverse, 5'GCCTTAAGAGCTGTAATTGAACTGGGAGTG-3'.

Antibodies. Antibody sources, species, dilutions, and references are provided in Supplemental Table 1.

Western blot. Immediately after harvesting kidneys, they were snap frozen in liquid nitrogen and stored at $-80^{\circ} \mathrm{C}$ until homogenization. They were homogenized using a Potter homogenizer in $1 \mathrm{ml}$ cold homogenization buffer containing lysis buffer that contained $300 \mathrm{mM}$ sucrose (Thermo Fisher Scientific), $50 \mathrm{mM}$ Tris- $\mathrm{HCl}$ (pH 7.4; MilliporeSigma), 1 mM EDTA (Thermo Fisher Scientific), 1 mM EGTA (MilliporeSigma), $1 \mathrm{mM} \mathrm{NaVO}_{4}$ (Calbiochem), $50 \mathrm{mM} \mathrm{NaF}$ (MilliporeSigma), $1 \mathrm{mM}$ ditiothreitol (MilliporeSigma), $1 \mathrm{mM}$ phenylmethane sulfonyl fluoride (MilliporeSigma), $1 \mathrm{mg} \mathrm{ml}^{-1}$ aprotinin (MilliporeSigma), and $4 \mathrm{mg}$ $\mathrm{ml}^{-1}$ leupeptin (Thermo Fisher Scientific) using a $10 \mathrm{ml}$ Potter-Elvehjem homogenizer. Cultured cells were scraped in RIPA buffer. Homogenates were centrifuged at $6,000 \mathrm{~g}$ for 15 minutes at $4^{\circ} \mathrm{C}$, and supernatants were transferred to a new tube and stored at $-80^{\circ} \mathrm{C}$. Then, $20 \mu \mathrm{g}$ protein were separated on a $4 \%-12 \%$ Bis-tris acetate gel (Invitrogen) and transferred to a polyvinylidene fluoride membrane by using Trans-Blot Turbo Transfer System (Bio-Rad). The membrane was blocked with 5\% nonfat milk in PBS-Tween (Thermo Fisher Scientific), followed by incubation with primary antibody for either 1 hour at room temperature or overnight at $4^{\circ} \mathrm{C}$. Membranes were washed, incubated with HRP-goat anti-rabbit IgG (1:7,500, Invitrogen, 65-6120) or HRP-rabbit anti-guinea pig IgG (1:7,500, Invitrogen, 61-4620), washed again, and finally incubated with Western Lightning ECL (Perkin Elmer). ECL signal was detected with a Syngene Pxi4 imager, and densitometry was performed with ImageJ (http://rsbweb.nih.gov/ij/). Protein abundance was normalized by densitometric quantitation of total protein via Coomassie staining (54). We also analyzed the Western blot data by normalizing to $\beta$-actin (Supplemental Figure 1). Protein abundance normalized by both methods led to the same conclusions. 
Primary renal epithelial cell culture and adenoviral infection. Primary renal epithelial cells were cultured in RPMI (without $\mathrm{Ca}^{2+}, \mathrm{Mg}^{2+}$ ) (Gibco) containing 10\% FBS (Thermo Fisher Scientific), 100 units/ml penicillin/streptomycin (Invitrogen), amphotericin B $(2.5 \mu \mathrm{g} / \mathrm{ml})$ (Thermo Fisher Scientific), EGF (20 ng/ml) (MilliporeSigma), L-glutamine (2 mM) (Gibco), and dexamethasone (50 nM) (Tocris Biosciences). Decapsulated whole kidneys were minced with a sterile scalpel and digested in $1 \mathrm{mg} / \mathrm{ml}$ collagenase type I (Worthington) in Ham's-F12 (MilliporeSigma) medium for 30 minutes. The digested kidney was then sieved through $100 \mu \mathrm{m}$ nylon mesh and the flow-through sieved again through $50 \mu$ m nylon mesh. The retained tubule pieces were then washed from the $50 \mu \mathrm{m}$ nylon mesh and centrifuged at $200 \mathrm{~g}$ for 5 minutes. Following one wash in Dulbecco's PBS (Gibco), oneninth of the tubule mix was plated per well in medium and left undisturbed for 72 hours before the first medium change. Once cells had grown out to $80 \%$ confluency, they were infected with empty adenovirus (Ad-Null) or Cre recombinase adenovirus (Ad-Cre) (both from Vector Biolabs) at a multiplicity of infection (MOI) of 20, assuming $2 \times 10^{6}$ cells per well. Cells were harvested in RIPA buffer 48 hours later.

$I F$. Animals were injected with anesthesia cocktail (ketamine/xylazine/acepromazine, 50/5/0.5 mg kg-1; Mylan, Llod Laboratories, and Boehringer Ingelheim Vetmedica, respectively), and under deep anesthesia, animals were perfusion fixed with 4\% paraformaldehyde (Thermo Fisher Scientific). After cryoprotection in 800 mOsmol sucrose (Thermo Fisher Scientific) and freezing in OCT (Sakura Finetek), 5 - $\mu \mathrm{m}$ sections were cut. For CUL3 IF, paraffin-embedded sections were used. Sections were incubated overnight at room temperature with primary antibodies (Supplemental Table 1). When double-staining was used with 2 primary antibodies raised in rabbit, after incubation with the first primary, sections were incubated with AffiniPure Fab Fragment goat antirabbit IgG (1:40, Jackson ImmunoResearch, 111-007-003) to permit its detection with anti-goat secondaries. Controls confirmed that the first primary was not detected by anti-rabbit secondary antibody (data not shown). Secondary antibodies used were Alexa Fluor 647 donkey anti-rabbit IgG (Invitrogen, A31573; 1:2,000), Alexa Fluor 488 donkey anti-rabbit IgG (Invitrogen, A21206, 1:2,000), Alexa Fluor 488 goat anti-guinea pig IgG (Invitrogen, A11073, 1:2,000), Alexa Fluor 488 donkey anti-goat IgG (Invitrogen, A11055, 1:2,000), Alexa Fluor 647 donkey anti-sheep IgG (Invitrogen, A21448, 1:1,000), and FITC-goat anti-mouse IgG (Zymed, 81-6511, 1:3,000), all incubated for 1 hour at room temperature. All sections were stained with DAPI in mounting medium (ProLong Diamond Antifade Mountant with DAPI, Thermo Fisher Scientific); some pictures were not merged with the picture from the DAPI channel. Images were captured with a ZEISS AXIO Imager M2 fluorescent microscope or a Zeiss LSM 880 confocal microscope.

Blood pressure measurement. Blood pressure was measured in male mice only by radio telemetry using PA-C10 transmitters (Data Sciences Inc.) implanted in the left carotid artery, with the transmitter inserted into a pocket in the body cavity, as previously described. Isoflurane ( $4 \%$ in $\mathrm{O}_{2}$ to induce, $1.5 \%-2 \%$ to maintain; Piramel Enterprises) was used to anesthetize the animals. Following surgery, mice received $0.1 \mathrm{mg} \mathrm{kg}^{-1}$ buprenorphine (s.c.; West-Ward Pharmaceuticals) analgesia twice daily for 3 days. Animals were monitored for alertness, activity, and loss of weight, and they were given at least 7 days to recover from surgery before measurements were recorded for 10 seconds every 10 minutes. Mean of the hourly averages were used to calculate mean SBP.

Blood analysis. Blood was collected via cardiac puncture under isoflurane anesthesia and transferred into heparinized tubes; $80 \mu 1$ was loaded into a Chem8+ cartridge for electrolyte measurement by i-STAT analyzer (Abbot Point of Care Inc.).

Amiloride response test. Animals were first injected i.p. with vehicle (0.09\% saline) and then placed in metabolic cages. Urine was collected for 6 hours. The next day, the same animals were injected with $40 \mu \mathrm{g}$ $25 \mathrm{~g}^{-1}$ body weight amiloride hydrochloride in vehicle, followed by a 6-hour urine collection. Urine $\mathrm{Na}^{+}$ and $\mathrm{K}^{+}$were determined by flame photometry.

Statistics. The null hypothesis was tested using 2-tailed unpaired $t$ tests, or 1-way ANOVA for repeated measures by using GraphPad Prism 7 as indicated in the figure legends. All data are plotted as means \pm SEM. $P<0.05$ was considered significant.

Study approval. Animal studies were performed in adherence to the NIH Guide for the Care and Use of Laboratory Animals (National Academies Press, 2011) and approved by the Oregon Health \& Science University IACUC (protocol IP00286).

\section{Author Contributions}

MZF and JAM designed the study. MZF, LNM, TS, and JAM conducted experiments and analyzed data. LNA and CDS provided the Loxp-STOP-Loxp-Cul3-49-IRES-tdTomato transgenic mouse model and assisted 
with study design and data interpretation. JDS provided reagents and assisted with study design and data interpretation. MZF and JAM wrote the manuscript, and all authors provided critical comments.

\section{Acknowledgments}

Funding for this project was provided by NIH grants R01DK098141 to JAM, R01HL125603 to CDS, and R01DK051496 to JDS; a fellowship from the German research Foundation (DFG - Forschungsstipendium; 332853055) to TS, and American Heart Association Postdoctoral Fellowship 17POST33670206 to MZF.

Address correspondence to: James A. McCormick; Division of Nephrology \& Hypertension, Department of Medicine, Oregon Health \& Science University, 2730 Moody Avenue, CL3NR, Portland, Oregon 97201, USA. Phone: 503.494.3980; Email: mccormij@ohsu.edu.

1. Hadchouel J, Ellison DH, Gamba G. Regulation of Renal Electrolyte Transport by WNK and SPAK-OSR1 Kinases. Annu Rev Physiol. 2016;78:367-389.

2. Huang CL, Yang SS, Lin SH. Mechanism of regulation of renal ion transport by WNK kinases. Curr Opin Nephrol Hypertens. 2008;17(5):519-525.

3. Louis-Dit-Picard H, et al. KLHL3 mutations cause familial hyperkalemic hypertension by impairing ion transport in the distal nephron. Nat Genet. 2012;44(4):456-460.

4. Boyden LM, et al. Mutations in kelch-like 3 and cullin 3 cause hypertension and electrolyte abnormalities. Nature. 2012;482(7383):98-102.

5. Terker AS, et al. Direct and Indirect Mineralocorticoid Effects Determine Distal Salt Transport. J Am Soc Nephrol. 2016;27(8):2436-2445.

6. Ferdaus MZ, et al. SPAK and OSR1 play essential roles in potassium homeostasis through actions on the distal convoluted tubule. J Physiol (Lond). 2016;594(17):4945-4966.

7. Pacheco-Alvarez $\mathrm{D}$, et al. The $\mathrm{Na}+\mathrm{Cl}$ - cotransporter is activated and phosphorylated at the amino-terminal domain upon intracellular chloride depletion. J Biol Chem. 2006;281(39):28755-28763.

8. Richardson $\mathrm{C}$, et al. Activation of the thiazide-sensitive Na+-Cl- cotransporter by the WNK-regulated kinases SPAK and OSR1. J Cell Sci. 2008;121(Pt 5):675-684.

9. Hoorn EJ, Nelson JH, McCormick JA, Ellison DH. The WNK kinase network regulating sodium, potassium, and blood pressure. J Am Soc Nephrol. 2011;22(4):605-614.

10. Ferdaus MZ, McCormick JA. The CUL3/KLHL3-WNK-SPAK/OSR1 pathway as a target for antihypertensive therapy. Am J Physiol Renal Physiol. 2016;310(11):F1389-F1396.

11. Rodan AR, Huang CL. Distal potassium handling based on flow modulation of maxi-K channel activity. Curr Opin Nephrol Hypertens. 2009;18(4):350-355.

12. Palmer LG, Schnermann J. Integrated control of Na transport along the nephron. Clin J Am Soc Nephrol. 2015;10(4):676-687.

13. Subramanya AR, Ellison DH. Distal convoluted tubule. Clin J Am Soc Nephrol. 2014;9(12):2147-2163.

14. Ellison DH, Terker AS, Gamba G. Potassium and Its Discontents: New Insight, New Treatments. J Am Soc Nephrol. 2016;27(4):981-989.

15. Pearce D, Soundararajan R, Trimpert C, Kashlan OB, Deen PM, Kohan DE. Collecting duct principal cell transport processes and their regulation. Clin J Am Soc Nephrol. 2015;10(1):135-146.

16. Pathare G, Hoenderop JG, Bindels RJ, San-Cristobal P. A molecular update on pseudohypoaldosteronism type II. Am J Physiol Renal Physiol. 2013;305(11):F1513-F1520.

17. Gormley K, Dong Y, Sagnella GA. Regulation of the epithelial sodium channel by accessory proteins. Biochem J. 2003;371(Pt 1):1-14

18. Muto S, Furuse M, Kusano E. Claudins and renal salt transport. Clin Exp Nephrol. 2012;16(1):61-67.

19. Nüsing RM, Pantalone F, Gröne HJ, Seyberth HW, Wegmann M. Expression of the potassium channel ROMK in adult and fetal human kidney. Histochem Cell Biol. 2005;123(6):553-559.

20. Vidal-Petiot E, et al. WNK1-related Familial Hyperkalemic Hypertension results from an increased expression of L-WNK1 specifically in the distal nephron. Proc Natl Acad Sci USA. 2013;110(35):14366-14371.

21. Petroski MD, Deshaies RJ. Function and regulation of cullin-RING ubiquitin ligases. Nat Rev Mol Cell Biol. 2005;6(1):9-20.

22. Bosu DR, Kipreos ET. Cullin-RING ubiquitin ligases: global regulation and activation cycles. Cell Div. $2008 ; 3: 7$.

23. Ohta A, et al. The CUL3-KLHL3 E3 ligase complex mutated in Gordon's hypertension syndrome interacts with and ubiquitylates WNK isoforms: disease-causing mutations in KLHL3 and WNK4 disrupt interaction. Biochem J. 2013;451(1):111-122.

24. Shibata S, Zhang J, Puthumana J, Stone KL, Lifton RP. Kelch-like 3 and Cullin 3 regulate electrolyte homeostasis via ubiquitination and degradation of WNK4. Proc Natl Acad Sci USA. 2013;110(19):7838-7843.

25. Susa K, et al. Impaired degradation of WNK1 and WNK4 kinases causes PHAII in mutant KLHL3 knock-in mice. Hum Mol Genet. 2014;23(19):5052-5060

26. Wakabayashi M, et al. Impaired KLHL3-mediated ubiquitination of WNK4 causes human hypertension. Cell Rep. 2013;3(3):858-868.

27. Sasaki E, et al. KLHL3 Knockout Mice Reveal the Physiological Role of KLHL3 and the Pathophysiology of Pseudohypoaldosteronism Type II Caused by Mutant KLHL3. Mol Cell Biol. 2017;37(7):e00508-16.

28. McCormick JA, et al. Hyperkalemic hypertension-associated cullin 3 promotes WNK signaling by degrading KLHL3. J Clin Invest. 2014;124(11):4723-4736.

29. Ibeawuchi SR, Agbor LN, Quelle FW, Sigmund CD. Hypertension-causing Mutations in Cullin 3 Protein Impair RhoA Protein Ubiquitination and Augment the Association with Substrate Adaptors. J Biol Chem. 2015;290(31):19208-19217. 
30. Schumacher FR, et al. Characterisation of the Cullin-3 mutation that causes a severe form of familial hypertension and hyperkalaemia. EMBO Mol Med. 2015;7(10):1285-1306.

31. Araki Y, et al. Generation and analysis of knock-in mice carrying pseudohypoaldosteronism type II-causing mutations in the cullin 3 gene. Biol Open. 2015;4(11):1509-1517.

32. Murthy M, Kurz T, O’Shaughnessy KM. WNK signalling pathways in blood pressure regulation. Cell Mol Life Sci. 2017;74(7):1261-1280

33. Traykova-Brauch M, et al. An efficient and versatile system for acute and chronic modulation of renal tubular function in transgenic mice. Nat Med. 2008;14(9):979-984.

34. Agbor LN, et al. Cullin-3 mutation causes arterial stiffness and hypertension through a vascular smooth muscle mechanism. JCI Insight. 2016;1(19):e91015.

35. Wimuttisuk W, Singer JD. The Cullin3 ubiquitin ligase functions as a Nedd8-bound heterodimer. Mol Biol Cell. 2007;18(3):899-909.

36. $\mathrm{Lin} \mathrm{SH}$, et al. Impaired phosphorylation of $\mathrm{Na}(+)-\mathrm{K}(+)-2 \mathrm{Cl}(-)$ cotransporter by oxidative stress-responsive kinase-1 deficiency manifests hypotension and Bartter-like syndrome. Proc Natl Acad Sci USA. 2011;108(42):17538-17543.

37. Richardson C, et al. Regulation of the NKCC2 ion cotransporter by SPAK-OSR1-dependent and -independent pathways. J Cell Sci. 2011;124(Pt 5):789-800.

38. Cheng CJ, Yoon J, Baum M, Huang CL. STE20/SPS1-related proline/alanine-rich kinase (SPAK) is critical for sodium reabsorption in isolated, perfused thick ascending limb. Am J Physiol Renal Physiol. 2015;308(5):F437-F443.

39. Ponce-Coria J, et al. A novel Ste20-related proline/alanine-rich kinase (SPAK)-independent pathway involving calcium-binding protein 39 (Cab39) and serine threonine kinase with no lysine member 4 (WNK4) in the activation of $\mathrm{Na}-\mathrm{K}-\mathrm{Cl}$ cotransporters. $J$ Biol Chem. 2014;289(25):17680-17688.

40. Ohno M, et al. Immunolocalization of WNK4 in mouse kidney. Histochem Cell Biol. 2011;136(1):25-35.

41. Kahle KT, et al. WNK4 regulates apical and basolateral Cl- flux in extrarenal epithelia. Proc Natl Acad Sci USA. 2004;101(7):2064-2069.

42. McCormick JA, et al. A SPAK isoform switch modulates renal salt transport and blood pressure. Cell Metab. 2011;14(3):352-364.

43. Terker AS, et al. Potassium modulates electrolyte balance and blood pressure through effects on distal cell voltage and chloride. Cell Metab. 2015;21(1):39-50.

44. Terker AS, Zhang C, Erspamer KJ, Gamba G, Yang CL, Ellison DH. Unique chloride-sensing properties of WNK4 permit the distal nephron to modulate potassium homeostasis. Kidney Int. 2016;89(1):127-134.

45. Chowdhury JA, Liu CH, Zuber AM, O’Shaughnessy KM. An inducible transgenic mouse model for familial hypertension with hyperkalaemia (Gordon's syndrome or pseudohypoaldosteronism type II). Clin Sci. 2013;124(12):701-708.

46. Hunter RW, Craigie E, Homer NZ, Mullins JJ, Bailey MA. Acute inhibition of NCC does not activate distal electrogenic Na+ reabsorption or kaliuresis. Am J Physiol Renal Physiol. 2014;306(4):F457-F467.

47. Grimm PR, Coleman R, Delpire E, Welling PA. Constitutively Active SPAK Causes Hyperkalemia by Activating NCC and Remodeling Distal Tubules. J Am Soc Nephrol. 2017;28(9):2597-2606.

48. McCormick JA, Ellison DH. Distal convoluted tubule. Compr Physiol. 2015;5(1):45-98.

49. Wilson FH, et al. Human hypertension caused by mutations in WNK kinases. Science. 2001;293(5532):1107-1112

50. Wen D, et al. Relation between BK- $\alpha / \beta 4$-mediated potassium secretion and ENaC-mediated sodium reabsorption. Kidney Int 2014;86(1):139-145

51. Wade JB, Liu J, Coleman R, Grimm PR, Delpire E, Welling PA. SPAK-mediated NCC regulation in response to low-K+ diet. Am J Physiol Renal Physiol. 2015;308(8):F923-F931.

52. Grimm PR, et al. SPAK isoforms and OSR1 regulate sodium-chloride co-transporters in a nephron-specific manner. $J$ Biol Chem. 2012;287(45):37673-37690.

53. McEvoy JD, Kossatz U, Malek N, Singer JD. Constitutive turnover of cyclin E by Cul3 maintains quiescence. Mol Cell Biol. 2007;27(10):3651-3666

54. McDonough AA, Veiras LC, Minas JN, Ralph DL. Considerations when quantitating protein abundance by immunoblot. Am J Physiol, Cell Physiol. 2015;308(6):C426-C433. 